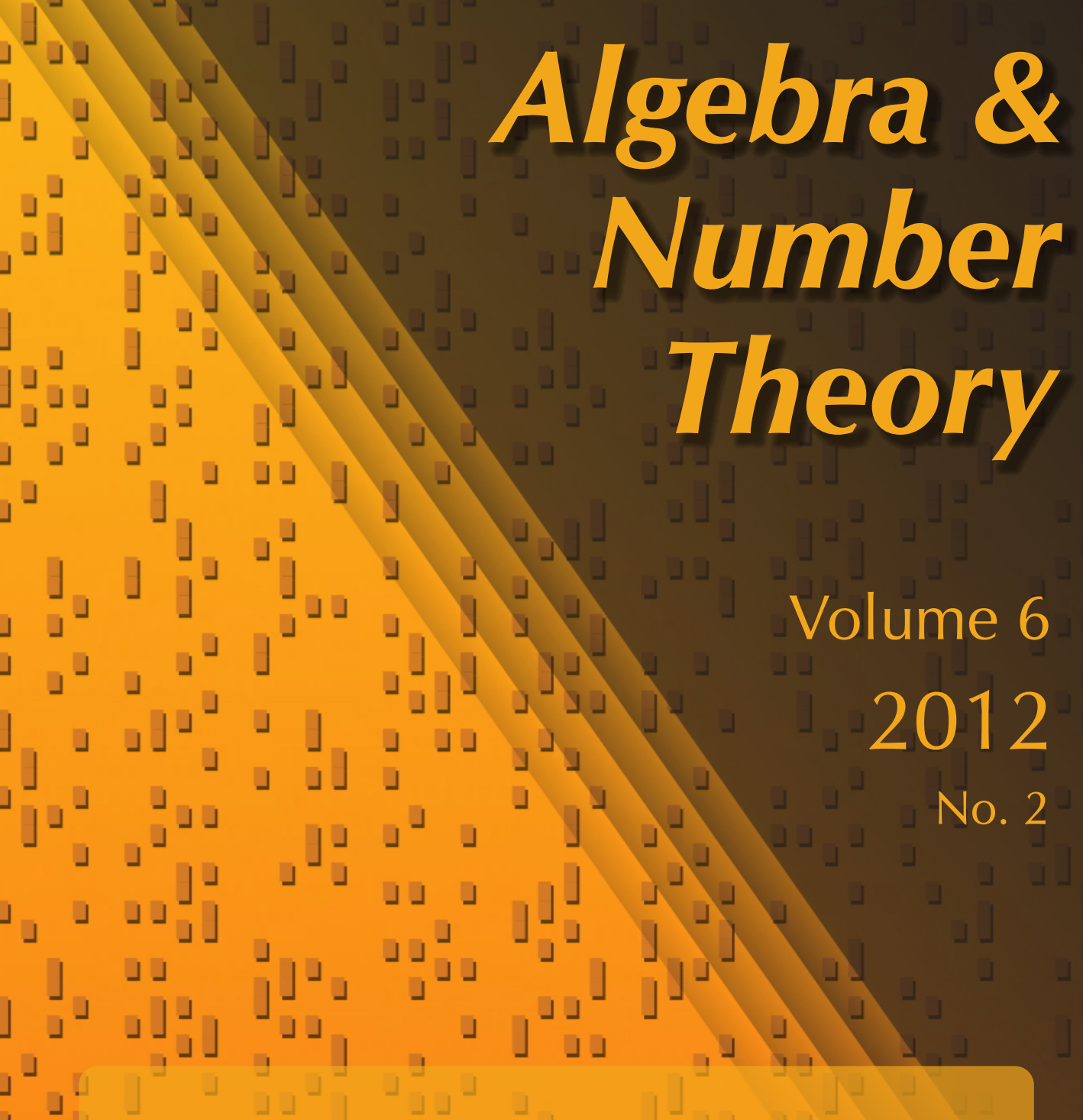

Moving lemma for additive higher Chow groups

\lrcorner Amalendu Krishna and Jinhyun Park

\lrcorner$\lrcorner$.

\lrcorner

\rfloor

\lrcorner$\lrcorner$

Volume 6
2012
No.

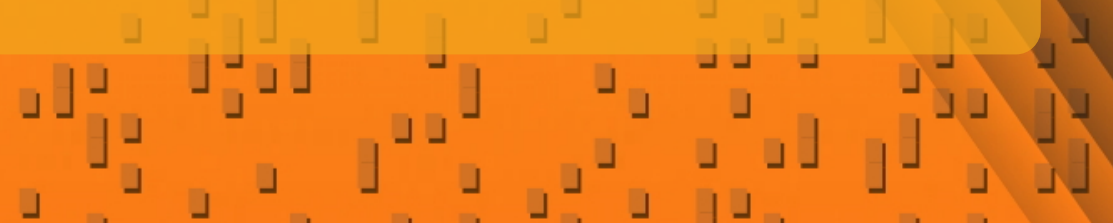

\lrcorner

J

\lrcorner

\lrcorner$\left.\left.\left.\left.\lrcorner\lrcorner\lrcorner\lrcorner\lrcorner\lrcorner\lrcorner\lrcorner\lrcorner_{\lrcorner}\right\lrcorner\right\lrcorner_{\lrcorner}\right\lrcorner\right\lrcorner$

صathematical sciences publishers $\lrcorner$ 


\title{
Moving lemma for additive higher Chow groups
}

\author{
Amalendu Krishna and Jinhyun Park
}

\begin{abstract}
We study additive higher Chow groups with several modulus conditions. Apart from exhibiting the validity of all known results for the additive Chow groups with these modulus conditions, we prove the moving lemma for them: for a smooth projective variety $X$ and a finite collection $\mathcal{W}$ of its locally closed algebraic subsets, every additive higher Chow cycle is congruent to an admissible cycle intersecting properly all members of $\mathcal{W}$ times faces. This is the additive analogue of the moving lemma for the higher Chow groups studied by S. Bloch and M. Levine.

As an application, we prove that any morphism from a quasiprojective variety to a smooth projective variety induces a pull-back map of additive higher Chow groups. More important applications of this moving lemma are derived in two separate papers by the authors.
\end{abstract}

\section{Introduction}

Working with algebraic cycles, formal finite sums of closed subvarieties of a variety, often requires some forms of moving results, as differential geometry often requires Sard's lemma. A classical example is Chow's moving lemma [1956], which moves algebraic cycles under rational equivalence. A modern version for higher Chow groups [Bloch 1994; Levine 1998] shows that, for a smooth quasiprojective variety $X$ and a finite set of locally closed subvarieties of $X$, one can move (modulo boundaries) admissible cycles to other admissible cycles that intersect a given finite set of subvarieties in the right codimensions. Any such result on moving of cycles is generally referred to as a moving lemma. Such moving results have played a very crucial role in the development and application of the theory of higher Chow groups. For instance, one major application was the construction of a triangulated category of mixed motives over $k$ [Hanamura 2004].

The primary goal of this paper is to prove this latter kind of moving lemma for additive higher Chow groups of a smooth and projective variety, which will

MSC2000: primary 14C25; secondary 19E15.

Keywords: Chow group, algebraic cycle, moving lemma. 
serve as an important technical tool in the study of additive higher Chow groups. Already, this moving lemma for additive Chow groups has been crucially used in [Krishna and Park 2011; 2012] for proving some important results about additive higher Chow groups. We expect that this will have many more applications in the future study of additive Chow groups and the infinitesimal $K$-theory of smooth varieties.

Additive Chow groups of 0-cycles on a field were first introduced in [Bloch and Esnault 2003b] in an attempt to describe the $K$-theory and motivic cohomology of the ring of dual numbers via algebraic cycles. Bloch and Esnault [2003a] later defined these groups by putting a modulus condition on additive Chow cycles in the hope of describing the $K$-groups of any given truncated polynomial ring over a field. The additive higher Chow groups of any given variety were defined in the most general form in [Park 2009] and were later studied in more detail in [Krishna and Levine 2008], where many nice properties of these groups were established.

The most crucial part of existing definitions of additive higher Chow groups, which makes them distinct from the higher Chow groups, is the modulus condition on the admissible additive cycles. This condition also brings an extra subtlety which does not appear in the theory of higher Chow groups. As conjectured in [Krishna and Levine 2008; Park 2009], additive higher Chow groups are expected to complement higher Chow groups for nonreduced schemes so as to obtain the right motivic cohomology groups. In particular, for a smooth projective variety $X$, one expects an Atiyah-Hirzebruch spectral sequence

$$
\mathrm{TH}^{-q}(X,-p-q ; m) \Rightarrow K_{-p-q}^{\mathrm{nil}}(X ; m),
$$

where $K^{\text {nil }}(X ; m)$ is the homotopy fiber of the restriction map

$$
K(X \times \operatorname{Spec}(k[t])) \rightarrow K\left(X \times \operatorname{Spec}\left(k[t] / t^{m+1}\right)\right) .
$$

Since these statements are still conjectural, it is not clear if the modulus conditions used to study additive higher Chow groups of varieties in the literature are the right ones to give the correct motivic cohomology, for example, ones which would satisfy (1-1). One goal of this paper is to exhibit that the modulus condition (which we call $M_{\text {sup }}$ in this paper) used in [Krishna and Levine 2008] may not be the best possible one.

We study the theory of additive Chow groups based on two other modulus conditions in this paper: $M=M_{\text {sum }}$ is based on the modulus condition used in [Bloch and Esnault 2003a; Rülling 2007], and $M=M_{\text {ssup }}$ is a new modulus condition introduced in this paper. Although this new modulus condition $M_{\text {ssup }}$ may appear to be mildly stronger than the one used in [Krishna and Levine 2008; Park 2009], it turns out that the resulting additive Chow groups have all the properties known for the additive Chow groups of [Bloch and Esnault 2003a; Krishna and Levine 
2008; Park 2009]. In addition, we prove many other crucial structural properties of additive higher Chow groups based on the modulus conditions $M_{\text {sum }}$ and $M_{\text {ssup }}$. More important properties are discussed in [Krishna and Park 2011; 2012].

As in the case of higher Chow groups, any theory of additive motivic cohomology which would compute the $K$-theory as in (1-1) is expected to have a form of moving lemma to make it more amenable to deeper study. The central result of the paper is the following moving lemma:

Theorem 4.1. For a smooth projective variety $X$ and a finite collection $W$ of its locally closed algebraic subsets, every additive higher Chow cycle is congruent to an admissible cycle intersecting properly all members of W times faces. In other words, the inclusion of complexes

$$
\mathrm{TZ}_{\mathscr{W}}^{q}(X, \cdot ; m) \hookrightarrow \mathrm{TZ}^{q}(X, \cdot ; m)
$$

is a quasiisomorphism.

This is the additive analogue of the moving lemma for the higher Chow groups studied by S. Bloch and M. Levine.

It is known that the moving lemma for all smooth quasiprojective varieties indirectly implies other properties such as $\mathbb{A}^{1}$-homotopy invariance and localization sequences. But these clearly fail for the additive Chow groups. This suggests that the above moving lemma may not be valid for some smooth quasiprojective varieties. A concrete quasiprojective example, where the standard arguments fail, is given in Example 8.2.

Our proof of the above result is broadly speaking based on the techniques of [Bloch 1986; Levine 1998] where the analogous result for the higher Chow groups is proven. However, the main difficulty with the techniques of both these works is that their arguments are mostly intersection theoretic and are not equipped to handle the more delicate modulus condition of additive Chow cycles. So these arguments cannot be directly transported to the additive world. This has made people believe that the additive Chow group may not satisfy the moving lemma.

We achieve the goal by our new containment-type argument (see Proposition 2.4) and construction of the additive version of a chain homotopy variety in Section 5. Using these results and Proposition 5.2, we show that we can keep track of the modulus condition whenever we need to move an additive cycle. On the log-additive higher Chow groups of [Krishna and Levine 2008], one can prove the moving lemma for any general smooth quasiprojective varieties using our main theorem.

As the first application of the moving lemma, we establish the contravariant functoriality property of the additive higher Chow groups in the most general form:

Theorem 7.1. For a morphism $f: X \rightarrow Y$ of quasiprojective varieties over a field $k$, where $Y$ is smooth and projective, there is a pull-back map 


$$
f^{*}: \mathrm{TH}^{q}(Y, n ; m) \rightarrow \mathrm{TH}^{q}(X, n ; m),
$$

and this satisfies the expected composition law.

If $X$ is also smooth and projective, the pull-back map on the additive Chow groups was constructed in [Krishna and Levine 2008] using the action of the higher Chow groups on the additive ones. However, the contravariant functoriality in this general form as above is new, and it is based on a crucial use of the moving lemma (Theorem 4.1) as in the case of general pull-back maps of higher Chow groups [Bloch 1986, Theorem 4.1], and another use of our containment argument to establish the Gysin chain map for regular embeddings. Even in the special case of $X$ being smooth and projective, our proof is different and more direct than the one in [Krishna and Levine 2008].

We give applications of the results in this paper elsewhere. In [Krishna and Park 2011] we investigate the structure of differential graded algebras on the additive higher Chow groups of smooth projective varieties. When $X=\operatorname{Spec}(k)$, this was done in [Rülling 2007]. Higher-dimensional varieties $X$ require involved calculations and arguments as well as the moving lemma and the containment lemma of this paper. As another application of the moving lemma, we showed in [Krishna and Park 2011] that there is an additive analogue of Bloch's normalized cycle complex and it is quasiisomorphic to the additive cycle complex. This fact is used to propose and study a motivic cyclic homology theory by constructing a mixed complex in the sense of A. Connes (see [Loday 1998]) from additive higher Chow complexes.

In [Krishna and Park 2012] we apply the moving lemma to construct a triangulated category $\mathscr{D} \mathcal{M}(k ; m)$ of mixed motives over $k[t] /\left(t^{m+1}\right)$. This category extends the category of [Hanamura 2004], and some "augmented motives" in the category compute the usual higher Chow groups and the additive higher Chow groups at the same time, as desired originally in [Bloch and Esnault 2003a, §4].

We now outline the structure of this paper. In Section 2, we define our basic objects, the additive higher Chow groups with various modulus conditions. We also prove some preliminary results used repeatedly in the paper. In Section 3, we prove basic properties of these additive Chow groups. In particular, we demonstrate, for the additive higher Chow groups based on the modulus condition $M_{\text {ssup }}$, all those results which are known for the additive higher Chow groups of [Bloch and Esnault 2003a; Krishna and Levine 2008; Park 2009] with slightly different modulus conditions $M_{\text {sum }}$ and $M_{\text {sup }}$. Section 4 gives the proofs of further preliminary results needed to prove our moving lemma for the additive higher Chow groups. The subsequent Sections 5 and 6 are devoted to our main result, the moving lemma for additive higher Chow groups. In Section 7, we apply the moving lemma to prove the general contravariant functoriality theorem, Theorem 7.1. In Section 8, 
we append some calculations of the additive higher Chow groups we found in the process of working on the problem. This suggests some kind of "pseudo"-A $\mathbb{A}^{1}$ homotopy properties of additive higher Chow groups.

Throughout this paper, a $k$-scheme, or a scheme over $k$, is always a separated scheme of finite type over a perfect field $k$. A $k$-variety is an integral $k$-scheme.

\section{Additive higher Chow groups}

In this section, we define additive higher Chow groups from a more unified perspective than those in the literature by Bloch and Esnault, Rülling, Krishna and Levine, and Park, treating the modulus conditions as "variables". We also prove some elementary results that are needed to study and compare additive Chow groups based on various modulus conditions.

We begin by fixing some notations which will be used throughout this paper. We write Sch $/ k, \mathbf{S m} / k$, and SmProj / $k$ for the categories of $k$-schemes, smooth quasiprojective varieties, and smooth projective varieties, respectively. We shall let $\mathbf{S} \mathbf{c h}^{\prime} / k$ denote the category of $k$-schemes with only proper maps. $D^{-}(\mathbf{A b})$ is the derived category of bounded-above complexes of abelian groups. Recall from [Krishna and Levine 2008; Park 2009] that for a normal variety $X$ over $k$, and a finite set of Weil divisors $\left\{Y_{1}, \ldots, Y_{s}\right\}$ on $X$, the supremum of these divisors, denoted by $\sup _{1 \leq i \leq s} Y_{i}$, is the Weil divisor defined to be

$$
\sup _{1 \leq i \leq s} Y_{i}=\operatorname{sum}_{Y \in \operatorname{Pdiv}(X)}\left(\max _{1 \leq i \leq s} \operatorname{ord}_{Y}\left(Y_{i}\right)\right)[Y],
$$

where $\operatorname{Pdiv}(X)$ is the set of all prime Weil divisors of $X$. One observes that the set of all Cartier divisors on a normal scheme $X$ is contained in the set of all Weil divisors, and the supremum of a collection of Cartier divisors may not remain a Cartier divisor in general, unless $X$ is factorial. We shall need some elementary results about Cartier and Weil divisors on normal varieties:

Lemma 2.1. Let $X$ be a normal variety and let $D_{1}$ and $D_{2}$ be effective Cartier divisors on $X$ such that $D_{1} \geq D_{2}$ as Weil divisors. Let $Y \subset X$ be a closed subset which intersects $D_{1}$ and $D_{2}$ properly. Let $f: Y^{N} \rightarrow X$ be the composite of the inclusion and the normalization of $Y_{\text {red. Then }} f^{*}\left(D_{1}\right) \geq f^{*}\left(D_{2}\right)$.

Proof. For any effective Cartier divisor $D$ on $X$, let $\Phi_{D}$ denote the sheaf of ideals defining $D$ as a locally principal closed subscheme of $X$. We first claim that $D_{1} \geq$ $D_{2}$ if and only if $\mathscr{I}_{D_{1}} \subset \mathscr{I}_{D_{2}}$. We only need to show the "only if" part, as the other implication is obvious. Now, $D_{1} \geq D_{2}$ implies that $D=D_{1}-D_{2}$ is effective as a Cartier divisor since the group of Cartier divisors forms a subgroup of Weil divisors on a normal scheme. Since $\mathscr{I}_{D_{1}} \subset \mathscr{I}_{D_{2}}$ is a local question, we can assume that $X=\operatorname{Spec}(A)$ is a local normal integral scheme and $\mathscr{I}_{D_{i}}=\left(a_{i}\right)$. Put $a=a_{1} / a_{2}$ 
as an element of the function field of $X$. We need to show that $a \in A$. Since $A$ is normal, it suffices to show that $a \in A_{\mathfrak{p}}$ for every height-one prime ideal $\mathfrak{p}$ of $A$. But this is precisely the meaning of $D_{1} \geq D_{2}$. This proves the claim.

Since $D_{i}$ intersect $Y$ properly, we see that $f^{*}\left(D_{i}\right)$ is a locally principal closed subscheme of $Y^{N}$ for $i=1,2$. The lemma now follows directly from the above claim.

The following is a refinement of [Krishna and Levine 2008, Lemma 3.2].

Lemma 2.2. Let $f: Y \rightarrow X$ be a surjective map of normal integral $k$-schemes. Let $D$ be a Cartier divisor on $X$ such that $f^{*}(D) \geq 0$ on $Y$. Then $D \geq 0$ on $X$.

Proof. As is implicit in the proof of Lemma 2.1, we can localize at the generic points of $\operatorname{Supp}(D)$ and assume that $X=\operatorname{Spec}(A)$, where $A$ is a discrete valuation ring which is essentially of finite type over $k$. The divisor $D$ is then given by a rational function $a \in K$, where $K$ is the field of fractions of $A$. Choosing a uniformization parameter $\pi$ of $A$, we can write $a$ uniquely as $a=u \pi^{n}$, where $u \in A^{\times}$and $n \in \mathbb{Z}$.

Since $f$ is surjective, there is a closed point $y \in Y$ such that $f(y)$ is the closed point of $X$. Since $Y$ is integral, the surjectivity of $f$ also implies that the generic point of $Y$ (which is also the generic point of $\operatorname{Spec}\left(\mathcal{O}_{Y, y}\right)$ ) must go to the generic point of $X$ under $f$. Hence the map $\operatorname{Spec}\left(\mathcal{O}_{Y, y}\right) \rightarrow X$ is surjective. This implies in particular that the image of $\pi$ in $O_{Y, y}$ is a nonzero element of the maximal ideal $\mathfrak{m}$ of the local ring $O_{Y, y}$. On the other hand, $f^{*}(D) \geq 0$ implies that as a rational function on $Y, a$ actually lies in $\mathscr{O}_{Y, y}$. Since $u \in \mathcal{O}_{Y, y}^{\times}$and $\pi \in \mathfrak{m}$, this can happen only when $n \geq 0$. That is, $D$ is effective.

We will assume that a $k$-scheme $X$ is equidimensional to define the additive Chow groups, although one can easily remove this condition by writing the additive Chow cycles in terms of their dimensions rather than their codimensions. Throughout this paper, for any such scheme $X$, we shall denote the normalization of $X_{\text {red }}$ by $X^{N}$. Thus $X^{N}$ is the disjoint union of the normalizations of all the irreducible components of $X_{\text {red }}$.

Set $\mathbb{A}^{1}:=\operatorname{Spec} k[t], \mathbb{G}_{m}:=\operatorname{Spec} k\left[t, t^{-1}\right], \mathbb{P}^{1}:=\operatorname{Proj} k\left[Y_{0}, Y_{1}\right]$, and let $y:=$ $Y_{1} / Y_{0}$ be the standard coordinate function on $\mathbb{P}^{1}$. We set $\square^{n}:=\left(\mathbb{P}^{1} \backslash\{1\}\right)^{n}$. For $n \geq 1$, let $B_{n}=\mathbb{G}_{m} \times \square^{n-1}, \widetilde{B}_{n}=\mathbb{A}^{1} \times \square^{n-1}, \bar{B}_{n}=\mathbb{A}^{1} \times\left(\mathbb{P}^{1}\right)^{n-1} \supset \widetilde{B}_{n}$, and $\widehat{B}_{n}=\mathbb{P}^{1} \times\left(\mathbb{P}^{1}\right)^{n-1} \supset \bar{B}_{n}$. We use the coordinate system $\left(t, y_{1}, \ldots, y_{n-1}\right)$ on $\widehat{B}_{n}$, with $y_{i}:=y \circ q_{i}$, where $q_{i}: \widehat{B}_{n} \rightarrow \mathbb{P}^{1}$ is the projection onto the $i$-th $\mathbb{P}^{1}$.

Let $F_{n, i}^{1}$, for $i=1, \ldots, n-1$, be the Cartier divisor on $\widehat{B}_{n}$ defined by $\left\{y_{i}=1\right\}$ and $F_{n, 0} \subset \widehat{B}_{n}$ the Cartier divisor defined by $\{t=0\}$. Notice that the divisor $F_{n, 0}$ is in fact contained in $\bar{B}_{n} \subset \widehat{B}_{n}$. Let $F_{n}^{1}$ denote the Cartier divisor $\operatorname{sum}_{i=1}^{n-1} F_{n, i}^{1}$ on $\widehat{B}_{n}$. 
A face of $B_{n}$ is a subscheme $F$ defined by equations of the form

$$
y_{i_{1}}=\epsilon_{1}, \quad \ldots, \quad y_{i_{s}}=\epsilon_{s} \quad\left(\epsilon_{j} \in\{0, \infty\}\right) .
$$

For $\epsilon=0, \infty$, and $i=1, \ldots, n-1$, let $\iota_{n, i, \epsilon}: B_{n-1} \rightarrow B_{n}$ be the inclusion

$$
\iota_{n, i, \epsilon}\left(t, y_{1}, \ldots, y_{n-2}\right)=\left(t, y_{1}, \ldots, y_{i-1}, \epsilon, y_{i}, \ldots, y_{n-2}\right) .
$$

We now define the modulus conditions that we shall consider for defining our additive higher Chow groups.

\section{A. Modulus conditions.}

Definition 2.3. Let $X$ be a $k$-scheme as above and let $V$ be an integral closed subscheme of $X \times B_{n}$. Let $\bar{V}$ denote the closure of $V$ in $X \times \widehat{B}_{n}$ and let

$$
v: \bar{V}^{N} \rightarrow X \times \widehat{B}_{n}
$$

denote the induced map from the normalization of $\bar{V}$. We fix an integer $m \geq 1$.

(1) We say that $V$ satisfies the modulus $m$ condition $M_{\text {sum }}$ (or the sum-modulus condition) on $X \times B_{n}$ if as Weil divisors on $\bar{V}^{N}$,

$$
(m+1)\left[v^{*}\left(F_{n, 0}\right)\right] \leq\left[v^{*}\left(F_{n}^{1}\right)\right] .
$$

This condition was used in [Bloch and Esnault 2003a; Rülling 2007] to study additive Chow groups of 0 -cycles on fields.

(2) We say that $V$ satisfies the modulus $m$ condition $M_{\text {sup }}$ (or the sup-modulus condition) on $X \times B_{n}$ if as Weil divisors on $\bar{V}^{N}$,

$$
(m+1)\left[v^{*}\left(F_{n, 0}\right)\right] \leq \sup _{1 \leq i \leq n-1}\left[v^{*}\left(F_{n, i}^{1}\right)\right] .
$$

This condition was used by in [Krishna and Levine 2008; Park 2009] to define their additive higher Chow groups.

(3) We say that $V$ satisfies the modulus $m$ condition $M_{\text {ssup }}$ (or the strong supmodulus condition) on $X \times B_{n}$ if there exists an integer $1 \leq i \leq n-1$ such that

$$
(m+1)\left[v^{*}\left(F_{n, 0}\right)\right] \leq\left[v^{*}\left(F_{n, i}^{1}\right)\right]
$$

as Weil divisors on $\bar{V}^{N}$.

Since the modulus conditions are defined for a given fixed integer $m$, we shall often simply say that $V$ satisfies a modulus condition $M$ without mentioning the integer $m$. Notice that since $V$ is contained in $X \times B_{n}$, its closure $\bar{V}$ intersects all the Cartier divisors $F_{n, 0}$ and $F_{n, i}^{1}(1 \leq i \leq n-1)$ properly in $X \times \widehat{B}_{n}$. In particular, 
their pull-backs of $F_{n, 0}$ and $F_{n, i}^{1}$ are all effective Cartier divisors on $\bar{V}^{N}$. Notice also that

$$
M_{\text {ssup }} \Rightarrow M_{\text {sup }} \Rightarrow M_{\text {sum }} \text {. }
$$

The following restriction property of the modulus conditions $M_{\text {sum }}$ and $M_{\text {ssup }}$ will be used repeatedly in this paper.

Proposition 2.4 (containment lemma). Let $X$ be a k-scheme, and let $W \subset V$ be irreducible closed subvarieties of $X \times B_{n}$. If $V$ satisfies $M_{\text {sum }}$, then so does $W$; if $V$ satisfies $M_{\text {ssup }}$, then so does $W$.

Proof. Let $\bar{V}$ and $\bar{W}$ be the Zariski closures of $V$ and $W$ in $X \times \widehat{B}_{n}$ and let $\bar{W} \stackrel{j}{\hookrightarrow} \bar{V}$ be the closed embedding. Let $\nu_{1}: \bar{V}^{N} \rightarrow \bar{V} \hookrightarrow X \times \widehat{B}_{n}$ be the normalization of $\bar{V}$ :

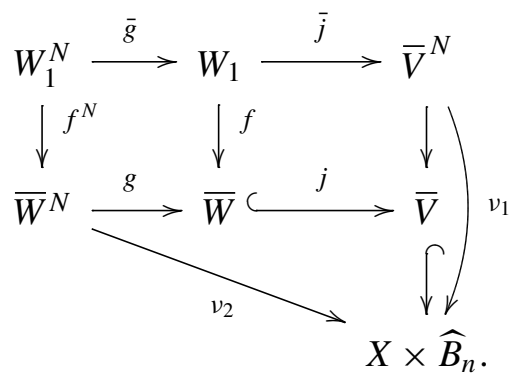

Let $W_{1}$ be $\bar{W} \times_{\bar{V}} \bar{V}^{N}$, and let $f$ and $\bar{j}$ be the natural projections. Let $g$ and $\bar{g}$ be the normalizations. The map $v_{2}$ is defined so that the lower triangle commutes. By the universal property of normalization, we have a finite surjective morphism $f^{N}: W_{1}^{N} \rightarrow \bar{W}^{N}$ of normal integral $k$-schemes that makes the above diagram commutative.

Since $V \cap F_{n, 0}=\varnothing$ and $W \neq \varnothing$, we see that $F_{n, 0}$ and $F_{n, i}^{1}$ intersect $\bar{W}$ properly. Now, if $V$ satisfies the modulus condition $M_{\text {ssup }}$, then Lemma 2.1 implies that there is an integer $1 \leq i \leq n-1$ such that $\bar{g}^{*} \circ \bar{j}^{*}\left[v_{1}^{*}\left(F_{n, i}^{1}-(m+1) F_{n, 0}\right)\right] \geq 0$ on $W_{1}^{N}$. In particular, by commutativity, we get $\left(f^{N}\right)^{*}\left[v_{2}^{*}\left(F_{n, i}^{1}-(m+1) F_{n, 0}\right)\right] \geq 0$ on $W_{1}^{N}$. Since $f^{N}$ is a finite and surjective map of normal varieties, from Lemma 2.2 we have $\left[v_{2}^{*}\left(F_{n, i}^{1}-(m+1) F_{n, 0}\right)\right] \geq 0$ on $\bar{W}^{N}$, that is, $W$ satisfies $M_{\text {ssup }}$ too.

The case of $M_{\text {sum }}$ follows exactly the same way using $F_{n}^{1}$ instead of $F_{n, i}^{1}$, noting that $F_{n}^{1}$ is also an effective Cartier divisor.

As one can see from the above proposition, although the modulus condition $M_{\text {sup }}$ lies between the other two modulus conditions $M_{\text {sum }}$ and $M_{\text {ssup }}$, the additive higher Chow groups based on the latter modulus conditions have better structural properties.

In this paper, we study the additive higher Chow groups based on the modulus conditions $M_{\text {sum }}$ and $M_{\text {ssup }}$. We shall show in the next section that the additive 
Chow groups based on our new modulus condition $M_{\text {ssup }}$ satisfy all the properties known to be satisfied by the additive higher Chow groups of Krishna and Levine, Park, Bloch and Esnault, and Rülling.

2B. Additive cycle complex. We define the additive cycle complex based on the above modulus conditions.

Definition 2.5. Let $M$ be a modulus condition - either $M_{\text {sum }}$ or $M_{\text {ssup }}$. Let $X$ be a $k$-scheme, and let $r$ and $m$ be integers with $m \geq 1$.

(0) $\mathrm{TZ}_{r}(X, 1 ; m)_{M}$ is the free abelian group on integral closed subschemes $Z$ of $X \times \mathbb{G}_{m}$ of dimension $r$.

For $n>1, \underline{\mathrm{TZ}}_{r}(X, n ; m)_{M}$ is the free abelian group on integral closed subschemes $Z$ of $X \times B_{n}$ of dimension $r+n-1$ such that:

(1) (Good position) For each face $F$ of $B_{n}, Z$ intersects $X \times F$ properly:

$$
\operatorname{dim}(Z \cap(X \times F)) \leq r+\operatorname{dim}(F)-1 \text {, and }
$$

(2) (Modulus condition) $Z$ satisfies the modulus $m$ condition $M$ on $X \times B_{n}$.

As our scheme $X$ is equidimensional of dimension $d$ over $k$, we write for $q \geq 0$

$$
\underline{\mathrm{TZ}}^{q}(X, n ; m)_{M}=\underline{\mathrm{TZ}}_{d+1-q}(X, n ; m)_{M} .
$$

We now observe that the good-position condition on $Z$ implies that the cycle $\left(\operatorname{id}_{X} \times l_{n, i, \epsilon}\right)^{*}(Z)$, that we denote by $\partial_{i}^{\epsilon}(Z)$, is well-defined and each component satisfies the good-position condition. Moreover, letting $Y=X \times F$ for $F=$ $\iota_{n, i, \epsilon}\left(B_{n-1}\right)$ in Proposition 2.4, we first of all see that $\bar{Y}$ intersects $X \times F_{n, 0}$ and $X \times F_{n}^{1}$ properly in $X \times \widehat{B}_{n}$, and each component of $\left(\operatorname{id}_{X} \times \iota_{n, i, \epsilon}\right)^{*}(Z)$ satisfies the modulus condition $M$ on $X \times B_{n-1}$. We thus conclude that if $Z \subset X \times B_{n}$ satisfies the above conditions (1) and (2), then every component of $\iota_{n, i, \epsilon}{ }^{*}(Z)$ also satisfies these conditions on $X \times B_{n-1}$. In particular, we have the cubical abelian group $\underline{n} \mapsto \underline{\mathrm{TZ}}^{q}(X, n ; m)_{M}$.

Definition 2.6. The additive cycle complex $\mathrm{TZ}^{q}(X, \cdot ; m)_{M}$ of $X$ in codimension $q$ and with modulus $m$ condition $M$ is the nondegenerate complex associated to the cubical abelian group $n \mapsto \underline{\mathrm{TZ}}^{q}(X, n ; m)_{M}$, that is,

$$
\mathrm{TZ}^{q}(X, n ; m)_{M}:=\frac{\underline{\mathrm{TZ}}^{q}(X, n ; m)_{M}}{\underline{\mathrm{TZ}}^{q}(X, n ; m)_{M, \operatorname{degn}}},
$$

where the group of degenerate cycles $\underline{\mathrm{TZ}}^{q}(X, n ; m)_{M \text {, degn }}$ is generated by the pullbacks of the cycles under the projections $X \times B_{n} \rightarrow X \times B_{n-1}$ given by

$$
\left(x, t, y_{1}, \ldots, y_{n-1}\right) \mapsto\left(x, t, y_{1}, \ldots, y_{i-1}, y_{i+1}, \ldots, y_{n-1}\right) .
$$


The boundary map of this complex at level $n$ is given by

$$
\partial=\operatorname{sum}_{i=1}^{n-1}(-1)^{i}\left(\partial_{i}^{\infty}-\partial_{i}^{0}\right),
$$

which satisfies $\partial^{2}=0$. The homology

$$
\mathrm{TH}^{q}(X, n ; m)_{M}:=H_{n}\left(\mathrm{TZ}^{q}(X, \cdot ; m)_{M}\right), \quad n \geq 1,
$$

is the additive higher Chow group of $X$ with modulus $m$ condition $M$.

From now on, we shall drop the subscript $M$ from the notations and it will be understood that the additive cycle complex or the additive higher Chow group in question is based on the modulus condition $M$, where $M$ could be either $M_{\text {sum }}$ or $M_{\text {ssup }}$. The reader should however always bear in mind that these two are different objects.

A few comments are in order. We could also have defined our additive cycle complex by taking $\mathrm{TZ}_{r}(X, n ; m)$ to be the free abelian group generated by integral closed subschemes of $X \times \widetilde{B}_{n}$ which have the good-intersection property with respect to the faces of $\widetilde{B}_{n}$, and which satisfy the modulus condition on $X \times \bar{B}_{n}$ (see [Krishna and Levine 2008; Park 2009]). However, the following easy consequence of the modulus condition shows that this does not change the cycle complex.

Lemma 2.7. Let $M$ be a modulus condition in Definition 2.3.

Then, there is a canonical bijection between the set of irreducible closed subvarieties $V \subset X \times B_{n}$ satisfying $M$ and the set of irreducible closed subvarieties $W \subset X \times \widetilde{B}_{n}$ satisfying $M$.

Here, the correspondence is actually given by the identity map. In other words, any closed subvariety satisfying $M$ on $X \times \widetilde{B}_{n}$ is in fact a closed subvariety of the smaller space $X \times B_{n}$.

Proof. First of all, since for any integral closed subscheme $V$ of $X \times \widehat{B}_{n}$, the pullback $v^{*}\left(F_{n, 0}\right)$ on $V^{N}$ is contained in the open subset $v^{-1}\left(X \times \bar{B}_{n}\right)$, we can replace $\widehat{B}_{n}$ by $\bar{B}_{n}$ in the definition of the modulus conditions.

Now, if $\Sigma$ and $\widetilde{\Sigma}$ are the two sets in the statement, then the modulus condition forces that if $V \in \Sigma$, then $V$ is the same as its closure in $X \times \widetilde{B}_{n}$. Conversely, if $V \in \widetilde{\Sigma}$, then the modulus condition again forces $V$ to be contained in $X \times B_{n}$.

Let $\mathrm{TZ}^{q}(X, \cdot ; m)_{\text {sup }}$ be the additive cycle complex as defined in [Krishna and Levine 2008; Park 2009]. This complex is based on the modulus condition $M_{\text {sup }}$ above. It follows from (2-3) that there are natural inclusions of cycle complexes

$$
\mathrm{TZ}^{q}(X, \cdot ; m)_{\text {ssup }} \hookrightarrow \mathrm{TZ}^{q}(X, \cdot ; m)_{\text {sup }} \hookrightarrow \mathrm{TZ}^{q}(X, \cdot ; m)_{\text {sum }}
$$

and hence there are natural maps

$$
\mathrm{TH}^{q}(X, \cdot ; m)_{\text {ssup }} \rightarrow \mathrm{TH}^{q}(X, \cdot ; m)_{\text {sup }} \rightarrow \mathrm{TH}^{q}(X, \cdot ; m)_{\text {sum }} .
$$


One drawback of the cycle complex based on $M_{\text {sup }}$ is that the underlying modulus condition for a cycle is not necessarily preserved when it is restricted to a face of $B_{n}$. This forces one to put an extra induction condition in the definition of $\mathrm{TZ}^{q}(X, \cdot ; m)_{\text {sup }}$ that requires for cycles to be admissible, not only must the cycles themselves satisfy $M_{\text {sup }}$ on $X \times B_{n}$, but also all their intersections with various faces

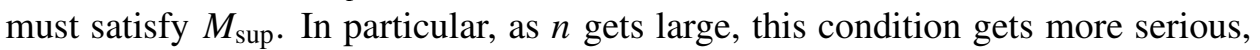
and it might be a very tedious job to find admissible cycles. On the other hand, the definition of our cycle complexes shows that this extra induction induction condition is superfluous for complexes based on $M_{\text {sum }}$ or $M_{\text {ssup }}$. Based on this discussion and all the results of this paper, one is led to guess that even though the modulus condition $M_{\text {ssup }}$ may appear mildly stronger (and $M_{\text {sum }}$ weaker) than the modulus condition $M_{\text {sup }}$, the following conjecture could be true.

Conjecture 2.8. For a smooth projective variety $X$ over $k$, the natural inclusions of cycle complexes $\mathrm{TZ}^{q}(X, \cdot ; m)_{\text {ssup }} \hookrightarrow \mathrm{TZ}^{q}(X, \cdot ; m)_{\text {sup }} \hookrightarrow \mathrm{TZ}^{q}(X, \cdot ; m)_{\text {sum }}$ are quasiisomorphisms.

In Section 3, combined with previously known results, we check that when $S=\operatorname{Spec}(k)$, for groups of 0-cycles, part of the conjecture holds, but we do not yet know how much of this conjecture holds true in general.

\section{Basic properties of $\mathbf{T H}^{q}(X, \cdot ; m)$}

In this section, our aim is to demonstrate that the additive higher Chow groups defined above for $M_{\text {sum }}$ and $M_{\text {ssup }}$ have all the properties (except Theorem 3.6 which we do not know for $M_{\text {sum }}$ ) which are known to be true for the additive Chow groups for $M_{\text {sup }}$ of [Krishna and Levine 2008; Park 2009]. Since most of the arguments in the proofs can be given either by quoting these references verbatim or by straightforward modifications of the same, we only give the sketches of the proofs with minimal explanations whenever deemed necessary. We begin with the following structural properties of our additive Chow groups.

Theorem 3.1. Let $f: Y \rightarrow X$ be a morphism of $k$-schemes.

(1) If $f$ is projective, there is a natural map of cycle complexes

$$
f_{*}: \mathrm{TZ}_{r}(Y, \cdot ; m) \rightarrow \mathrm{TZ}_{r}(X, \cdot ; m)
$$

that induces the analogous push-forward map on the homology.

(2) If $f$ is flat, there is a natural map of cycle complexes

$$
f^{*}: \mathrm{TZ}_{r}(X, \cdot ; m) \rightarrow \mathrm{TZ}_{r}(Y, \cdot ; m)
$$

that induces the analogous pull-back map on the homology. These pull-back and push-forward maps satisfy the obvious functorial properties. 
(3) If $X$ is smooth and projective, there is a product

$$
\cap_{X}: \mathrm{CH}^{r}(X, p) \otimes \mathrm{TH}_{s}(X, q ; m) \rightarrow \mathrm{TH}_{s-r}(X, p+q ; m),
$$

natural with respect to flat pull-back, that satisfies the projection formula

$$
f_{*}\left(f^{*}(a) \cap_{X} b\right)=a \cap_{Y} f_{*}(b)
$$

for $f: X \rightarrow Y$ a morphism of smooth projective varieties. If $f$ is flat in addition, we have an additional projection formula:

$$
f_{*}\left(a \cap_{X} f^{*}(b)\right)=f_{*}(a) \cap_{Y} b .
$$

(4) If $X$ is smooth and quasiprojective, there is a product

$$
\cap_{X}: \mathrm{CH}^{r}(X) \otimes \mathrm{TH}_{s}(X, q ; m) \rightarrow \mathrm{TH}_{s-r}(X, q ; m),
$$

natural with respect to flat pull-back, that satisfies the projection formula

$$
f_{*}\left(f^{*}(a) \cap_{X} b\right)=a \cap_{Y} f_{*}(b)
$$

for $f: X \rightarrow Y$ a projective morphism of smooth quasiprojective varieties. If $f$ is flat in addition, we have an additional projection formula

$$
f_{*}\left(a \cap_{X} f^{*}(b)\right)=f_{*}(a) \cap_{Y} b .
$$

Furthermore, all products are associative.

Proof. This follows from the arguments in [Krishna and Levine 2008]. Granting the flat pull-back and the projective push-forward, the theorem is a direct consequence of Lemmas 4.7 and 4.9 of that article, whose proofs are independent of the choice of the modulus conditions of Definition 2.3, as the interested reader may verify. The proofs of the flat pull-back and projective push-forward maps on the level of cycle complexes also follow in the same way as in [Krishna and Levine 2008] using our Lemma 2.2.

Theorem 3.2 (projective bundle and blow-up formulae). Let $X$ be a smooth quasiprojective variety and let $V$ be a vector bundle on $X$ of rank $r+1$. Let $p: \mathbb{P}(V) \rightarrow X$ be the associated projective bundle over $X$. Let $\eta \in \mathrm{CH}^{1}(\mathbb{P}(V))$ be the class of the tautological line bundle $\mathcal{O}(1)$. Then for any $q, n \geq 1$ and $m \geq 2$, the map

$$
\theta: \bigoplus_{i=0}^{r} \mathrm{TH}^{q-i}(X, n ; m) \rightarrow \mathrm{TH}^{q}(\mathbb{P}(V), n ; m)
$$

given by

$$
\left(a_{0}, \ldots, a_{r}\right) \mapsto \operatorname{sum}_{i=0}^{r} \eta^{i} \cap \mathbb{P}(V) p^{*}\left(a_{i}\right)
$$

is an isomorphism. 
Suppose that $i: Z \rightarrow X$ is a closed immersion of smooth projective varieties and $\mu: X_{Z} \rightarrow X$ is the blow-up of $X$ along $Z$ with $i_{E}: E \rightarrow X_{Z}$ the exceptional divisor with morphism $q: E \rightarrow Z$. Then the sequence

$0 \rightarrow \mathrm{TH}^{s}(X, n ; m) \stackrel{\left(i^{*}, \mu^{*}\right)}{\longrightarrow} \mathrm{TH}^{s}(Z, n ; m) \oplus \mathrm{TH}^{s}\left(X_{Z}, n ; m\right) \stackrel{q^{*}-i_{E}^{*}}{\longrightarrow} \mathrm{TH}^{s}(E, n ; m) \rightarrow 0$

is split exact.

Proof. In view of Theorem 3.1, the proof of the theorem is exactly the same as the proofs of [Krishna and Levine 2008, Theorems 5.6 and 5.8]. The basic point is that there is a similar decomposition of the motives of the projective bundle and the blow-up in the triangulated category $\operatorname{Mot}_{k}$ of motives over $k$ [ibid., Section 2]. On the other hand, Theorem 3.1 implies that for each integer $p \geq 1$, the assignment $(X, n) \mapsto \mathrm{TH}^{n}(X, p ; m)$ is a functor from $\mathrm{Mot}_{k}$ to the category of graded abelian groups for any modulus $M$. We refer the reader to [ibid., Section 5] for details.

Recall from [ibid., Section 2.4] that $K^{b}(\mathbb{Z} \mathbf{S m P r o j} / k)$ is the homotopy category of the bounded complexes in the additive category $\mathbb{Z} \mathbf{S m P r o j} / k$ generated by SmProj $/ k$. We denote the complex concentrated in degree 0 associated to an $X \in \mathbf{S m P r o j} / k$ by $[X]$. Sending $X$ to $[X]$ defines the functor

$$
[-]: \text { SmProj } / k \rightarrow K^{b}(\mathbb{Z} \text { SmProj } / k) .
$$

Let $i: Z \rightarrow X$ be a closed immersion in SmProj / $k, \mu: X_{Z} \rightarrow X$ the blow-up of $X$ along $Z$, and $i_{E}: E \rightarrow X_{Z}$ the exceptional divisor with structure morphism $q: E \rightarrow Z$. Let $C(\mu)$ be the complex

$$
[E] \stackrel{\left(i_{E},-q\right)}{\longrightarrow}\left[X_{Z}\right] \oplus[Z] \stackrel{\mu+i}{\longrightarrow}[X]
$$

with $[X]$ in degree 0 . The category $\mathscr{D}_{\text {hom }}(k)$ is the localization of the triangulated category $K^{b}(\mathbb{Z}$ SmProj $/ k)$ with respect to the thick subcategory generated by the complexes $C(\mu)$.

Theorem 3.3. Assume that $k$ admits resolution of singularities. Then the functor $\mathrm{TZ}_{r}(-; m): \mathbf{S m P r o j} / k \rightarrow D^{-}(\mathbf{A b})$ extends to a functor

$$
\mathrm{TZ}_{r}^{\log }(-; m): \mathbf{S c h} / k \rightarrow D^{-}(\mathbf{A b})
$$

together with a natural transformation of functors $\mathrm{TZ}_{r}^{\log }(-; m) \rightarrow \mathrm{TZ}_{r}(-; m)$ satisfying:

(1) Let $\mu: Y \rightarrow X$ be a proper morphism in $\mathbf{S c h} / k, i: Z \rightarrow X$ a closed immersion. Suppose that $\mu: \mu^{-1}(X \backslash Z) \rightarrow X \backslash Z$ is an isomorphism. Set $E:=\mu^{-1}(Z)$ with maps $i_{E}: E \rightarrow Y, q: E \rightarrow Z$. There is a canonical extension of the sequence in 
$D^{-}(\mathbf{A b})$ :

$$
\mathrm{TZ}_{r}^{\log }(E ; m) \stackrel{\left(i_{E *},-q_{*}\right)}{\longrightarrow} \mathrm{TZ}_{r}^{\log }(Y ; m) \oplus \mathrm{TZ}_{r}^{\log }(Z ; m) \stackrel{\mu_{*}+i_{*}}{\longrightarrow} \mathrm{TZ}_{r}^{\log }(X ; m)
$$

to a distinguished triangle in $D^{-}(\mathbf{A b})$.

(2) Let $i: Z \rightarrow X$ be a closed immersion in $\mathbf{S c h} / k, j: U \rightarrow X$ the open complement. Then there is a canonical distinguished triangle in $D^{-}(\mathbf{A b})$ :

$$
\mathrm{TZ}_{r}^{\log }(Z ; m) \stackrel{i_{*}}{\rightarrow} \mathrm{TZ}_{r}^{\log }(X ; m) \stackrel{j^{*}}{\rightarrow} \mathrm{TZ}_{r}^{\log }(U ; m) \rightarrow \mathrm{TZ}_{r}^{\log }(Z ; m)[1],
$$

which is natural with respect to proper morphisms of pairs $(X, U) \rightarrow\left(X^{\prime}, U^{\prime}\right)$.

(3) For any $X \in \mathbf{S c h} / k$, the natural map $\mathrm{TH}_{r}^{\log }(X, n ; m) \rightarrow \mathrm{TH}_{r+p}^{\log }\left(X \times \mathbb{A}^{p}, n ; m\right)$ is an isomorphism.

Proof. The proof of this theorem is exactly the same as the proof of [Krishna and Levine 2008, Corollary 6.2]. By [ibid., Lemma 2.8], the motive functor

$$
m_{\text {hom }}: \text { SmProj } / k \rightarrow \mathscr{D}_{\text {hom }}(k)
$$

is a category of homological descent in the sense of [Guillén and Navarro Aznar 2002]. Theorem 3.2 immediately implies that $\mathrm{TZ}_{r}(-; m): \mathbf{S m P r o j} / k \rightarrow D^{-}(\mathbf{A b})$ extends to a functor $\mathrm{TZ}_{r}(-; m): \mathscr{D}_{\text {hom }}(k) \rightarrow D^{-}(\mathbf{A b})$. On the other hand, the functor $m_{\text {hom }}$ extends to a functor $M_{\text {hom }}: \mathbf{S c h}^{\prime} / k \rightarrow \mathscr{D}_{\text {hom }}(k)$ by [Krishna and Levine 2008, Theorem 2.9]. The functor $\mathrm{TZ}_{r}^{\log }(-; m)$ is the composite $\mathrm{TZ}_{r}(-; m) \circ$ $M_{\text {hom }}$. All the desired properties of $\mathrm{TZ}_{r}^{\log }(-; m)$ follow from the similar properties of $M_{\mathrm{hom}}$ as shown in the same reference.

Next we study the question of the existence of the regulator maps from our additive higher Chow groups to the modules of absolute Kähler differentials. First we prove the following result of [Bloch and Esnault 2003a; Rülling 2007] on 0-cycles for the modulus condition $M_{\text {ssup }}$.

Theorem 3.4. Assume that char $(k) \neq 2$ and let $\mathbb{W}_{m} \Omega_{k}^{\bullet}$ denote the generalized de Rham-Witt complex of Hesselholt and Madsen (see [Rülling 2007]). Then there is a natural isomorphism

$$
R_{0, m}^{n}: \mathrm{TH}^{n}(k, n ; m) \rightarrow \mathbb{W}_{m} \Omega_{k}^{n-1} .
$$

Proof. This is already known for $M_{\text {sum }}$. For the modulus condition $M_{\text {ssup }}$, we first note that the map $R_{0, m}^{n}$ is the composite map

$$
\mathrm{TH}^{n}(k, n ; m)_{\text {ssup }} \rightarrow \mathrm{TH}^{n}(k, n ; m)_{\text {sum }} \stackrel{\theta}{\rightarrow} \mathbb{W}_{m} \Omega_{k}^{n-1},
$$

where $\theta$ is constructed in [Rülling 2007] and this coincides with the regulator map of Bloch and Esnault for $m=1$. Furthermore for $m=1$, Bloch and Esnault define 
the inverse map $\Omega_{k}^{n-1} \rightarrow \mathrm{TH}^{n}(k, n ; 1)_{\text {sum }}$ using a presentation of $\Omega_{k}^{n-1}$. The reader can easily check from the proof of [Bloch and Esnault 2003a, Proposition 6.3] that the inverse map is actually defined from $\Omega_{k}^{n-1}$ to $\operatorname{TH}^{n}(k, n ; 1)_{\text {ssup. This completes }}$ the proof when $m=1$.

For $m \geq 2$, Rülling's proof for $\mathrm{TH}^{n}(k, n ; 1)_{\text {sum }}$ has these main steps:

(1) The existence of map $R_{0, m}^{n}$

(2) The isomorphism of $R_{0, m}^{1}$.

(3) The existence of transfer maps on the additive higher Chow groups for finite extensions of fields.

(4) Showing that pro-group $\left\{\mathrm{TH}^{n}(k, n ; m)\right\}_{n, m \geq 1}$ is an example of a restricted Witt complex; see [Rülling 2007, Remark 4.22].

We have already shown (1) for our $\mathrm{TH}^{n}(k, n ; m)_{\text {sup }}$. The proof of (3) is a simple consequence of Theorem 3.1. The surjectivity part of (2) follows from the result of Rülling and the isomorphism $\underline{\mathrm{TZ}}^{n}(k, n ; m)_{\text {ssup }}=\underline{\mathrm{TZ}}^{n}(k, n ; m)_{\text {sum }}$. To prove injectivity, we follow the proof of [Rülling 2007, Corollary 4.6.1] and observe that if there is a cycle $\zeta \in \underline{\mathrm{TZ}}^{1}(k, 1 ; m)$ such that $R_{0, m}^{1}(\zeta)=0$, then $\zeta$ is the boundary of a curve $C$ which is an admissible cycle with the modulus condition $M_{\text {sum }}$. But then $C$ is an admissible cycle also with the modulus condition $M_{\text {ssup }}$ since one has $M_{\text {ssup }}=M_{\text {sup }}=M_{\text {sum }}$ when $n=2$ by definition. This proves (2). Note that this does not need any assumptions on the characteristic of the ground field.

For the proof of (4), one checks that Lemma 4.17 of [ibid.] works without change.

Rülling showed that these four ingredients and the universality of the de RhamWitt complex imply that there is a map

$$
\mathbb{W}_{m} \Omega_{k}^{n-1} \stackrel{S_{0, m}^{n}}{\longrightarrow} \mathrm{TH}^{n}(k, n ; m)
$$

which is surjective. On the other hand, one checks from the construction of the map $R_{0 . m}^{n}$ in [ibid.] that $R_{0 . m}^{n} \circ S_{0 . m}^{n}$ is the identity.

The following result is an immediate consequence of the results of Rülling and Theorem 3.4. This gives evidence for Conjecture 2.8.

Corollary 3.5. For every $n, m \geq 1$, the natural maps

$$
\mathrm{TH}^{n}(k, n ; m)_{\text {ssup }} \rightarrow \mathrm{TH}^{n}(k, n ; m)_{\text {sup }} \rightarrow \mathrm{TH}^{n}(k, n ; m)_{\text {sum }}
$$

are isomorphisms.

We finally turn to the regulator maps for 1-cycles as considered in [Park 2009]. 
Theorem 3.6. Suppose that $k$ is of characteristic zero and assume the modulus

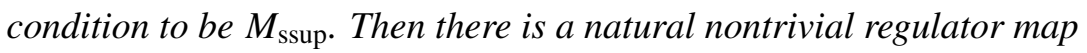

$$
R_{1, m}^{n}: \mathrm{TH}^{n-1}(k, n ; m)_{\text {ssup }} \rightarrow \Omega_{k}^{n-3} .
$$

This map is surjective if $k$ is, moreover, algebraically closed.

Proof. Let $R_{1, m}^{n}$ be the composite map

$$
\mathrm{TH}^{n}(k, n ; m)_{\text {ssup }} \rightarrow \mathrm{TH}^{n}(k, n ; m)_{\text {sup }} \stackrel{\theta}{\rightarrow} \Omega_{k}^{n-3},
$$

where $\theta$ is constructed in [Park 2009]. For the nontriviality of $R_{1, m}^{n}$, Park constructs a 1-cycle $\Gamma$ (see [Park 2007, Proposition 1.9] and [Krishna and Levine 2008, 7.11]) and shows (see [Park 2007, Lemmas 1.7 and 1.9]) that each component of $\Gamma$ in

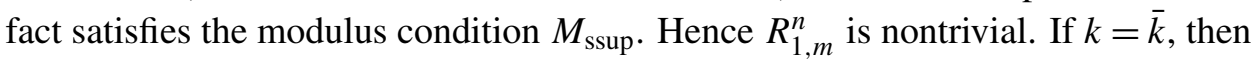
the proof of the surjectivity in [Krishna and Levine 2008, §7] follows from the following:

(1) An action of $k^{\times}$on $\mathrm{TH}^{n}(k, n ; m)$,

(2) Suitable $k^{\times}$-equivariance of $R_{1, m}^{3}$ up to a scalar,

(3) The surjectivity of $R_{1, m}^{3}$, and

(4) The cap product $\mathrm{CH}^{n}(k, n) \otimes_{\mathbb{Z}} \mathrm{TH}^{2}(k, 3 ; m) \rightarrow \mathrm{TH}^{n+2}(k, n+3 ; m)$.

The action of $k^{\times}$on our additive higher Chow groups is given as in [Park 2007; Krishna and Levine 2008] by

$$
a *\left(x, t_{1}, \ldots, t_{n-1}\right)=\left(x / a, t_{1}, \ldots, t_{n-1}\right) .
$$

This action extends to an action of $k^{\times}$on $\widehat{B}_{n}$. The proof of (2) now follows from the $k^{\times}$-equivariance of the natural map $\mathrm{TZ}_{r}(k, n ; m)_{\text {ssup }} \rightarrow \mathrm{TZ}_{r}(k, n ; m)_{\text {sup }}$ and the results of [Krishna and Levine 2008]. The proof of (3) is a direct consequence of (1), (2), and the fact that $k$ is algebraically closed field of characteristic zero. Finally, (4) is already shown in Theorem 3.1.

We do not yet know if this theorem holds for $M_{\text {sum }}$ because the regulator map $R_{1, m}^{n}$ in [Park 2007] is not immediately defined on the set of all $M_{\text {sum-admissible }}$ 1-cycles. In fact, this was one main obstruction that led to the introduction of the $M_{\text {sup }}$ modulus condition in that work. See Section 8 A for a related discussion on how one may potentially get around this issue.

\section{Preliminaries for moving lemma}

The underlying additive cycle complexes and additive higher Chow groups in all the results in the rest of this paper will be based on the modulus condition $M_{\text {sum }}$ or 
$M_{\text {ssup }}$, unless one of these is specifically mentioned. Our next three sections will be devoted to proving our first main result of this paper:

Theorem 4.1. Let $X$ be a smooth projective variety over a perfect field $k$. Let $\mathfrak{W}$ be a finite collection of locally closed subsets of $X$. Then, the inclusion of additive higher Chow cycle complexes (see below for definitions)

$$
\mathrm{TZ}_{\mathscr{W}}^{q}(X, \cdot ; m) \hookrightarrow \mathrm{TZ}^{q}(X, \cdot ; m)
$$

is a quasiisomorphism. In other words, every admissible additive higher Chow cycle is congruent to another admissible cycle intersecting properly all given finitely many locally closed subsets of $X$ times faces.

In this section, we set up our notations and machinery that are needed to prove this theorem, and prove some preliminary steps. Let $X$ be a smooth projective variety over $k$ and we fix an integer $m \geq 1$. Let $\mathscr{W}$ be a finite collection of locally closed algebraic subsets of $X$. If a member of $\mathscr{W}$ is not irreducible, we always replace it by all of its irreducible components so that we assume all members of $W$ are irreducible. For a locally closed subset $Y \subset X$, recall that the codimension $\operatorname{codim}_{X} Y$ is defined to be the minimum of $\operatorname{codim}_{X} Z$ for all irreducible components $Z$ of $Y$.

Definition 4.2. We define $\underline{\mathrm{TZ}}_{\mathscr{W}}^{q}(X, n ; m)$ to be the subgroup of $\underline{\mathrm{TZ}}^{q}(X, n ; m)$ generated by integral closed subschemes $Z \subset X \times B_{n}$ such that

(1) $Z$ is in $\underline{\mathrm{TZ}}^{q}(X, n ; m)$ and

(2) $\operatorname{codim}_{W \times F}(Z \cap(W \times F)) \geq q$ for all $W \in W$ and all faces $F$ of $B_{n}$.

It is easy to see that $\underline{\mathrm{TZ}}_{\mathscr{W}}^{q}(X, \cdot ; m)$ forms a cubical subgroup of $\underline{\mathrm{TZ}}^{q}(X, \cdot ; m)$, giving us the subcomplex

$$
\mathrm{TZ}_{\mathscr{W}}^{q}(X, \cdot ; m)=\frac{\underline{\mathrm{TZ}}_{\mathscr{W}}^{q}(X, \cdot ; m)}{\underline{\mathrm{TZ}}_{\mathscr{W}}^{q}(X, \cdot ; m)_{\operatorname{degn}}} \subset \mathrm{TZ}^{q}(X, \cdot ; m) .
$$

Let $\mathrm{TH}_{\mathscr{W}}^{q}(X, \cdot ; m)$ denote the homology of the complex $\mathrm{TZ}_{\mathscr{W}}^{q}(X, \cdot ; m)$. Then the above inclusion induces a natural map of homology,

$$
\mathrm{TH}_{\mathscr{W}}^{q}(X, \cdot ; m) \rightarrow \mathrm{TH}^{q}(X, \cdot ; m) .
$$

More generally, if $e: \mathcal{W} \rightarrow \mathbb{Z}_{\geq 0}$ is a set-theoretic function, then one can define subcomplexes $\underline{\mathrm{TZ}}_{W, e}^{q}(X, \cdot ; m)$ replacing condition (2) above by

(2e) $\operatorname{codim}_{W \times F}(Z \cap(W \times F)) \geq q-e(W)$.

In this generality, the subcomplex $\underline{\mathrm{TZ}}_{\mathscr{W}}^{q}(X, \cdot ; m)$ is the same as $\underline{\mathrm{TZ}}_{\mathscr{W}, 0}^{q}(X, \cdot ; m)$. 
Remark 4.3. Let $\Phi$ be the set of all set-theoretic functions $e: \mathscr{W} \rightarrow \mathbb{Z}_{\geq 0}$. Give a partial ordering on $\Phi$ by declaring $e^{\prime} \geq e$ if $e^{\prime}(W) \geq e(W)$ for all $W \in \mathscr{W}$. If two functions $e, e^{\prime} \in \Phi$ satisfy $e^{\prime} \geq e$, then for any irreducible admissible subvariety $Z \in \mathrm{TZ}_{\mathscr{W}, e}^{q}(X, n ; m)$, we have

$$
\operatorname{codim}_{W \times F}(Z \cap(W \times F)) \geq q-e(W) \geq q-e^{\prime}(W)
$$

for all $W \in \mathscr{W}$ and all faces $F \subset B_{n}$. Thus, we have

$$
\mathrm{TZ}_{\mathscr{W}, e}^{q}(X, n ; m) \subset \mathrm{TZ}_{\mathscr{W}, e^{\prime}}^{q}(X, n ; m) \quad \text { for } e \leq e^{\prime} .
$$

Note that if $e \in \Phi$ satisfies $e \geq q$ where $q$ is considered as a constant function in $\Phi$, then automatically

$$
\mathrm{TZ}_{\mathscr{W}, q}^{q}(X, n ; m)=\mathrm{TZ}_{\mathscr{W}, e}^{q}(X, n ; m)=\mathrm{TZ}^{q}(X, n ; m) .
$$

Since $0 \leq e$ for all $e \in \Phi$, for each triple $e, e^{\prime}, e^{\prime \prime}$ such that $e \leq e^{\prime} \leq q \leq e^{\prime \prime}$, we have

$$
\begin{aligned}
\mathrm{TZ}_{W}^{q}(X, n ; m) & \subset \mathrm{TZ}_{W, e}^{q}(X, n ; m) \subset \mathrm{TZ}_{W, e^{\prime}}^{q}(X, n ; m) \\
& \subset \mathrm{TZ}_{W, q}^{q}(X, n ; m)=\mathrm{TZ}_{W, e^{\prime \prime}}^{q}(X, n ; m)=\mathrm{TZ}^{q}(X, n ; m) .
\end{aligned}
$$

All these (in)equalities are equivariant with respect to the boundary maps.

Remark 4.4. The main theorem is equivalent to saying that the inclusion

$$
\mathrm{TZ}_{\mathscr{W}}^{q}(X, n ; m) \subset \mathrm{TZ}^{q}(X, n ; m)
$$

induces an isomorphism $\mathrm{TH}_{\mathscr{W}}^{q}(X, n ; m) \simeq \mathrm{TH}^{q}(X, n ; m)$ for the given modulus condition $M$.

Our remaining objective in this section is to prove an additive analogue of the spreading argument, which originates from Bloch's arguments. We begin with the following results.

Lemma 4.5. Let $f: X \rightarrow Y$ be a dominant morphism of integral normal varieties and let $\eta$ denote the generic point of $Y$. Consider the fiber diagram

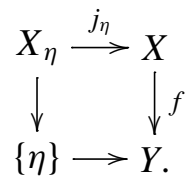

Let $D$ be a Weil divisor on $X$ such that $j_{\eta}^{*}(D)$ is effective. Then there is a nonempty open subset $U \subset Y$ such that if $j: f^{-1}(U) \rightarrow X$ denotes the open inclusion, then $j^{*}(D)$ is also effective. 
Proof. Let $D=\operatorname{sum} n_{i} D_{i}$. Then $j_{\eta}^{*}(D)$ is effective if and only if for every $i$ with $n_{i}<0$, one has $D_{i} \cap X_{\eta}=\varnothing$. Since $D$ is a finite sum, it suffices to show that if $D$ is a prime divisor on $X$ such that $D \cap X_{\eta}=\varnothing$, then there is a nonempty open subset $U \subset Y$ such that $D \cap f^{-1}(U)=\varnothing$.

Our hypothesis implies that $\overline{f(D)}$ is a proper closed subset of $Y$. Thus $U=$ $Y \backslash \overline{f(D)}$ is the desired open subset of $Y$.

Lemma 4.6. Let $X$ be a quasiprojective $k$-variety and let $\mathcal{W}$ be a finite collection of locally closed subsets of $X$. Let $K$ be a finite field extension of $k$. Let $X_{K}$ be the base extension $X_{K}=X \times_{\operatorname{Spec}(k)} \operatorname{Spec}(K)$, and let ${ }^{W_{K}}$ be the set of the base extensions of the varieties in $\mathcal{W}$. Then there are natural maps

$$
\begin{gathered}
p^{*}: \frac{\mathrm{TZ}^{q}(X, \cdot ; m)}{\mathrm{TZ}_{\mathscr{W}}^{q}(X, \cdot ; m)} \rightarrow \frac{\mathrm{TZ}^{q}\left(X_{K}, \cdot ; m\right)}{\mathrm{TZ}_{W_{K}}^{q}\left(X_{K}, \cdot ; m\right)}, \\
p_{*}: \frac{\mathrm{TZ}^{q}\left(X_{K}, \cdot ; m\right)}{\mathrm{TZ}_{W_{K}}^{q}\left(X_{K}, \cdot ; m\right)} \rightarrow \frac{\mathrm{TZ}^{q}(X, \cdot ; m)}{\mathrm{TZ}_{\mathscr{W}}^{q}(X, \cdot ; m)}
\end{gathered}
$$

such that $p_{*} \circ p^{*}=[K: k] \cdot i d$.

Proof. By Theorem 3.1, one also has the flat pull-back and finite push-forward maps $\mathrm{TZ}_{W^{\prime}}^{q}(X, \cdot ; m) \rightarrow \mathrm{TZ}_{W^{\prime} K}^{q}\left(X_{K}, \cdot ; m\right)$ and $\mathrm{TZ}_{W^{\prime} K}^{q}\left(X_{K}, \cdot ; m\right) \rightarrow \mathrm{TZ}_{W^{\prime}}^{q}(X, \cdot ; m)$ for any $\mathcal{W}^{\prime}$. Taking for $\mathcal{W}^{\prime}$ the collection $\{X\}$ and then $\mathcal{W}$, and then taking the quotient of the two, we get the desired maps. The last property of the composite map is obvious from the construction of the pull-back and the push-forward maps on the additive cycle complexes; see [Krishna and Levine 2008].

Proposition 4.7 (spreading lemma). Let $k \subset K$ be a purely transcendental extension. For a smooth projective variety $X$ over $k$ and any finite collection $W$ of locally closed algebraic subsets of $X$, let $X_{K}$ and $\mathcal{W}_{K}$ be the base extensions as before. Let $p_{K}: X_{K} \rightarrow X_{k}$ be the natural map. Then, the pull-back map

$$
p_{K}^{*}: \frac{\mathrm{TZ}^{q}(X, \cdot ; m)}{\mathrm{TZ}_{W}^{q}(X, \cdot ; m)} \rightarrow \frac{\mathrm{TZ}^{q}\left(X_{K}, \cdot ; m\right)}{\mathrm{TZ}_{W_{K}}^{q}\left(X_{K}, \cdot ; m\right)}
$$

is injective on homology.

Proof. First, suppose the proposition holds for all infinite fields, and let $k$ be a finite field. Let $Z$ be a cycle on the left quotient group whose pull-back via $k \rightarrow K$ dies. Then, for two different primes $\ell_{1}$ and $\ell_{2}$ and for pro- $\ell_{i}$ extensions $k \rightarrow k_{i}$, the images of $Z$ under the respective pull-backs are zero. Hence, by the norm argument in Lemma 4.6, there exist integers $N_{i}$ such that $\ell_{i}^{N_{i}} Z=0$ in the left group. This implies that $Z=0$, thus the proposition holds for the finite field $k$. Hence, we can assume that $k$ is infinite.

Since the additive Chow group of $X_{K}$ is an inductive limit of the additive Chow groups of $X_{L}$, where $L \subset K$ range over purely transcendental extension of $k$ of 
finite transcendence degree over $k$, we can assume that the transcendence degree of $K$ over $k$ is finite.

Now let $Z \in \mathrm{TZ}^{q}(X, n ; m)$ be a cycle such that $\partial Z \in \mathrm{TZ}_{\mathscr{W}}^{q}(X, n-1 ; m)$ where there are admissible cycles $B_{K} \in \mathrm{TZ}^{q}\left(X_{K}, n+1 ; m\right)$ and $V_{K} \in \mathrm{TZ}_{\mathscr{W}_{K}}^{q}\left(X_{K}, n ; m\right)$ satisfying $Z_{K}=\partial\left(B_{K}\right)+V_{K}$.

We first consider the natural inclusion of complexes

$$
\mathrm{TZ}^{q}(X, \cdot ; m) \hookrightarrow z^{q}\left(X \times \mathbb{A}_{k}^{1}, \cdot-1\right) .
$$

Since $K$ is the function field of some affine space $\mathbb{A}_{k}^{r}$, we can use the specialization argument for Bloch's cycle complexes [1986, Lemma 2.3] to find an open subset $U^{\prime} \subset \mathbb{A}_{k}^{r}$ and cycles

$$
B_{U^{\prime}} \in z^{q}\left(X \times U^{\prime} \times \mathbb{A}_{k}^{1}, n\right), \quad V_{U^{\prime}} \in z_{\mathscr{W} \times U^{\prime} \times \mathbb{A}_{k}^{1}}^{q}\left(X \times U^{\prime} \times \mathbb{A}_{k}^{1}, n-1\right)
$$

such that $B_{K}$ and $V_{K}$ are the restrictions of $B_{U^{\prime}}$ and $V_{U^{\prime}}$ to the generic point of $U^{\prime}$ and $Z \times U^{\prime}=\partial\left(B_{U^{\prime}}\right)+V_{U^{\prime}}$, respectively. In particular, all components of $B_{U^{\prime}}$ and $V_{U^{\prime}}$ intersect all faces of $X \times U^{\prime} \times B_{n+1}$ and $X \times U^{\prime} \times B_{n}$ properly. To make $B_{U^{\prime}}$ and $V_{U^{\prime}}$ admissible additive cycles, we modify them using our Lemma 4.5.

To check the modulus condition for our cycles, let $\eta$ denote the generic point $\operatorname{Spec}(K)$ of $U^{\prime}$. Let $\widehat{B}_{U^{\prime}}^{N}$ and $\widehat{V}_{U^{\prime}}^{N}$ denote the normalizations of the closures of $B_{U^{\prime}}$ and $V_{U^{\prime}}$ in $X \times U^{\prime} \times \widehat{B}_{n+1}$ and $X \times U^{\prime} \times \widehat{B}_{n}$, respectively.

We first prove the admissibility under the modulus condition $M_{\text {ssup }}$ which is a priori more difficult than $M_{\text {sum }}$. The admissibility of $B_{K}$ and $V_{K}$ implies that there are integers $1 \leq i \leq n$ and $1 \leq i^{\prime} \leq n-1$ such that in (4-5), the Weil divisors $j_{\eta}^{*}\left(F_{n+1, i}^{1}-(m+1) F_{n+1,0}\right)$ and $j_{\eta}^{*}\left(F_{n, i^{\prime}}^{1}-(m+1) F_{n, 0}\right)$ are effective on $\widehat{B}_{U^{\prime}, \eta}^{N}$ and $\widehat{V}_{U^{\prime}, \eta}^{N}$, respectively. Since $X$ and $\widehat{B}_{n}$ are projective, the maps $\widehat{B}_{U^{\prime}}^{N}, \widehat{V}_{U^{\prime}}^{N} \rightarrow U^{\prime}$ are projective. These maps are dominant since $B_{K}$ and $V_{K}$ are nonzero-cycles. Thus we can apply Lemma 4.5 to find an open subset $U \subset U^{\prime}$ such that

$$
j_{U}^{*}\left(F_{n+1, i}^{1}-(m+1) F_{n+1,0}\right)
$$

and $j_{U}^{*}\left(F_{n, i^{\prime}}^{1}-(m+1) F_{n, 0}\right)$ are also effective. The same argument applies for the modulus condition $M_{\text {sum }}$ as well. We just have to replace the Cartier divisors $F_{n+1, i}^{1}$ and $F_{n, i^{\prime}}^{1}$ by $F_{n+1}^{1}$ and $F_{n}^{1}$, respectively. Lemma 4.5 applies in this case, too.

Replacing $U^{\prime}$ by $U$, we see that

$$
\begin{gathered}
B_{U} \in \mathrm{TZ}^{q}(X \times U, n+1 ; m), \quad V_{U} \in \mathrm{TZ}_{\mathscr{W} \times U}^{q}(X \times U, n ; m), \\
Z \times U=\partial\left(B_{U}\right)+V_{U} .
\end{gathered}
$$

Next, (4-6) implies that for a $k$-rational point $u \in U(k)$ (which exists because $k$ is infinite) such that the restrictions of $B_{U}$ and $V_{U}$ to $X \times\{u\}$ give well-defined cycles in $z^{q}\left(X \times \mathbb{A}^{1}, n\right)$ and $z_{W \times \mathbb{A}_{k}^{1}}^{q}\left(X \times \mathbb{A}_{k}^{1}, n-1\right)$, one has $Z=\partial\left(i_{u}^{*}\left(B_{U}\right)\right)+i_{u}^{*}\left(V_{U}\right)$, where $i_{u}: X \times\{u\} \rightarrow X \times U$ is the closed immersion. 
We now only need to show that $i_{u}^{*}\left(B_{U}\right)$ and $i_{u}^{*}\left(V_{U}\right)$ satisfy the modulus condition on $X \times\{u\}$. But this follows directly from (4-6) and the containment lemma, Proposition 2.4.

\section{Moving lemma for projective spaces}

We follow the strategy of Bloch and Levine to prove the moving lemma for the additive higher Chow groups. This involves proving the moving lemma first for the projective spaces and then deducing the same for general smooth projective varieties using the techniques of linear projections. This section is devoted to the proof of the moving lemma for the projective spaces. We use the following technique from [Bloch 1986, Lemma 1.1] a few times to prove the proper-intersection properties of moved cycles with the prescribed algebraic sets.

Lemma 5.1. Let $X$ be an algebraic $k$-scheme and $G$ a connected algebraic $k$-group acting on $X$. Let $A, B \subset X$ be closed subsets, and assume that the fibers of the map

$$
G \times A \rightarrow X \quad(g, a) \mapsto g \cdot a
$$

all have the same dimension and that this map is dominant. Then, there exists a nonempty open subset $U \subset G$ such that for all extension fields $L$ of $k$ and for all $g \in U(L)$, the intersection $g\left(A_{L}\right) \cap B_{L}$ is proper in $X_{L}$.

Proposition 5.2 (admissibility of projective image). Let $f: X \rightarrow Y$ be a projective morphism of quasiprojective varieties over a field $k$. Let $Z \in \underline{\mathrm{TZ}}^{r}(X, n ; m)$ be an irreducible admissible cycle and let $V=f(Z)$. Then $V \in \underline{\mathrm{TZ}}^{s}(Y, n ; m)$, where $s$ is the codimension of $V$ in $Y \times B_{n}$.

Proof. We prove this in several steps.

Claim 1. $V$ intersects all codimension-one faces $F$ of $B_{n}$ properly in $B_{n}$.

Consider $F=F_{n, i}^{\epsilon}=\iota_{n, i, \epsilon}\left(B_{n-1}\right)$ for some $i \in\{1,2, \ldots, n-1\}, \epsilon \in\{0, \infty\}$, and consider the diagram

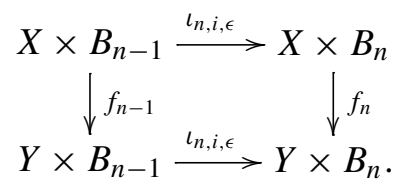

Since $F$ is a divisor in $B_{n}$, that $V$ intersects $Y \times F$ properly is equivalent to that $Y \times F \not \supset V$. Towards contradiction, suppose that $V \subset Y \times F$. Then,

$$
Z \subset f_{n}^{-1}\left(f_{n}(Z)\right)=f_{n}^{-1}(V) \subset f_{n}^{-1}(Y \times F)=\iota_{n, i, \epsilon}\left(f_{n-1}^{-1}\left(Y \times B_{n-1}\right)=X \times F .\right.
$$

By assumption, $Z$ intersects $X \times F$ properly so that we must have $Z \not \subset X \times F$. This contradiction proves the claim. 
Claim 2. $V$ intersects all lower-dimensional faces of $B_{n}$ properly.

By the admissibility assumption, all cycles $\partial_{i}^{\epsilon}(Z)=Z \cap\left(X \times F_{n, i}^{\epsilon}\right)$ are admissible. Moreover, it is easy to see that $\partial_{i}^{\epsilon}(V)=f_{n-1}\left(\partial_{i}^{\epsilon}(Z)\right)$. Thus we can replace $Z$ by $\partial_{i}^{\epsilon}(Z)$ and apply the same argument as above; inductively we see that $V$ has the good-intersection property.

Claim 3. For each face $F$ of $B_{n}$, including the case $F=B_{n}$, the cycle $V \cap(Y \times F)$ has the modulus condition.

For any face $F=\iota\left(B_{i}\right) \subset B_{n}$, where $\iota: B_{i} \hookrightarrow B_{n}$ is a face map, and for the projections $f_{i}: X \times B_{i} \rightarrow Y \times B_{i}$, note that $V \cap(Y \times F)=f_{n}(Z \cap(X \times F))=$ $f_{i}\left(\left.Z\right|_{X \times F}\right)$. But the admissibility of $Z$ implies that $\left.Z\right|_{X \times F}$ is also admissible (see Proposition 2.4). Hence, replacing $\left.Z\right|_{X \times F}$ by $Z$, we only need to prove it for $F=B_{n}$, that is, we just need to show that $V$ satisfies the modulus condition. Consider the diagram

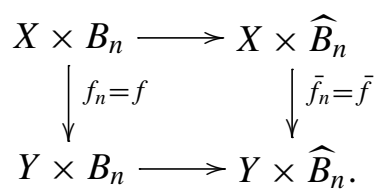

Subclaim. Let $\bar{V}$ be the closure of $V$ in $Y \times \widehat{B}_{n}$ and let $\bar{Z}$ be the closure of $Z$ in $X \times \widehat{B}_{n}$. Then $\bar{V}=\bar{f}(\bar{Z})$.

Since $Z \subset f^{-1}(V) \subset \bar{f}^{-1}(\bar{V})$ and $V$ is closed, we have $\bar{Z} \subset \bar{f}^{-1}(\bar{V})$. Hence, $\bar{f}(\bar{Z}) \subset \bar{V}$. For the other inclusion, note that $V=f(Z) \subset \bar{f}(\bar{Z})$ and $\bar{f}(\bar{Z})$ is closed because $\bar{f}$ is projective. Hence $\bar{V} \subset \bar{f}(\bar{Z})$. This proves this subclaim.

To prove the modulus condition for $V$, we take the normalizations $\nu_{\bar{Z}}: \bar{Z}^{N} \rightarrow \bar{Z}$ and $\nu_{\bar{V}}: \bar{V}^{N} \rightarrow \bar{V}$ of $\bar{Z}$ and $\bar{V}$, and consider the following diagram:

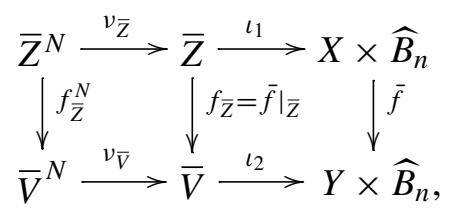

where $\iota_{1}$ and $\iota_{2}$ are the inclusions, and $f_{Z}^{N}$ is given by the universal property of the normalization $\nu_{\bar{V}}$ for dominant morphisms. Note that $f_{\bar{Z}}^{N}$ is automatically projective and surjective because $f_{Z}$ is so. Let $q_{\bar{Z}}:=\iota_{1} \circ \nu_{\bar{Z}}$ and $q_{\bar{V}}=\iota_{2} \circ v_{\bar{V}}$.

Suppose $Z$ satisfies the modulus condition $M_{\text {ssup }}$ and consider on $\widehat{B}_{n}$ the Cartier divisors $D_{i}:=F_{n, i}^{1}-(m+1) F_{n, 0}$ for $1 \leq i \leq n-1$. That the cycle $Z$ has the modulus condition means that $\left[q_{Z}^{*} \circ \bar{f}^{*}\left(D_{i}\right)\right] \geq 0$ for an index $i$. By the commutativity of the above diagram, this means that the Cartier divisor $f_{\bar{Z}}^{N *}\left[q_{\bar{V}}^{*}\left(D_{i}\right)\right] \geq 0$. By Lemma 2.2, this implies that $\left[q_{\bar{Z}}^{*}\left(D_{i}\right)\right] \geq 0$, which is the modulus condition for $V$. 
If $Z$ satisfies the modulus condition $M_{\text {sum }}$, we use the same argument by replacing $F_{n, i}^{1}$ with $F_{n}^{1}$. This finishes the proof of the proposition.

Remark 5.3. In Proposition 5.2, if $X$ is projective, $Y=\operatorname{Spec}(k)$, and $n=1$, then $V$ is always a single point. To see this, let $Z \subset X \times B_{1}=X \times \mathbb{G}_{m}$ be an admissible irreducible closed subvariety. Let $V=p(Z)$, where $p: X \times \mathbb{G}_{m} \rightarrow \mathbb{G}_{m}$ is the projection.

Since $X$ is complete, $p$ is a closed map. Hence, $V=p(Z)$ is an irreducible closed subvariety of $\mathbb{G}_{m}$. But the only closed subvarieties of $\mathbb{G}_{m}$ are finite subsets or all of $\mathbb{G}_{m}$. On the other hand, if $\bar{Z}$ is the closure of $Z$ in $X \times \mathbb{A}^{1}$, then the modulus condition implies that $\bar{Z} \cap|X \times\{t=0\}|=\varnothing$. This implies that $V$ must be a proper subset and hence a finite subset. Since $V$ is irreducible, consequently $V$ must be a nonzero single point.

Hence $Z=W \times\{*\}$ for a closed subvariety $W \subset X$, and a closed point $\{*\} \in \mathbb{G}_{m}$. Conversely, any such variety is admissible. This classifies all admissible cycles $Z$ when $X$ is projective and $n=1$.

For $n>1$, all we can say is that $Z$ is contained in $X \times V$, where $V$ is admissible in $\mathrm{TZ}_{s}(k, n ; m)$ for a suitable $s$.

5A. Homotopy variety. Now we want to construct the "homotopy variety". First, we need the following simple result:

Lemma 5.4. Let $\mathrm{SL}_{r+1, k}$ be the $(r+1) \times(r+1)$ special linear group over $k$, and let $\eta$ be the generic point of the $k$-variety $\mathrm{SL}_{r+1, k}$. Let $K$ be its function field (this is a purely transcendental extension of $k$ ). Let $\mathrm{SL}_{r+1, K}:=\mathrm{SL}_{r+1, k} \otimes_{k} K$ be base change. Then, there is a morphism of $K$-varieties $\phi: \square_{K}^{1} \rightarrow \mathrm{SL}_{r+1, K}$ such that $\phi(0)$ is the identity element, and $\phi(\infty)$ is the generic point $\eta$ considered as a K-rational point.

Proof. By a general result on the special linear groups, every element of $\mathrm{SL}_{r+1, K}$ is generated by the transvections $E_{i j}(a), i \neq j, a \in K$, that are $(r+1) \times(r+1)$ matrices where the diagonal entries are 1 , the $(i, j)$-entry is $a$ and all other entries are zero.

For each pair $(i, j)$, the collection $\left\{E_{i j}(a) \mid a \in K\right\}$ forms a one-parameter subgroup of $\mathrm{SL}_{r+1, K}$ isomorphic to $\mathbb{G}_{a, K}$. Thus, for each fixed $b \in K$, define $\phi_{i j}^{b}: \mathbb{A}_{K}^{1} \rightarrow \mathrm{SL}_{r+1, K}$ by $\phi_{i j}^{b}(y):=E_{i j}(b y)$.

Express the $K$-rational point $\eta$ of $\mathrm{SL}_{r+1, K}$ as the (ordered) product

$$
\eta=\prod_{l=1}^{p} E_{i_{l} j_{l}}\left(a_{l}\right), \quad \text { for some } i_{l}, j_{l} \in\{1,2, \ldots, r+1\} \text { and } a_{l} \in K
$$


and define $\phi^{\prime}: \mathbb{A}_{K}^{1} \rightarrow \mathrm{SL}_{r+1, K}$ by $\phi^{\prime}=\prod_{l=1}^{p} \phi_{i_{l} j_{l}}^{a_{l}}$. By definition, we have $\phi^{\prime}(0)=\mathrm{Id}$ and $\phi^{\prime}(1)=\eta$. Composing with the automorphism $\sigma: \mathbb{P}_{K}^{1} \rightarrow \mathbb{P}_{K}^{1}$ given by

$$
y \mapsto \frac{y}{y-1},
$$

which isomorphically maps $\square_{K}^{1}$ to $\mathbb{A}_{K}^{1}$, we obtain $\phi=\phi^{\prime} \circ \sigma: \square_{K}^{1} \rightarrow \mathrm{SL}_{r+1, K}$. This $\phi$ satisfies the desired properties.

Recall that one consequence of Lemma 2.7 is that the additive cycle complex with modulus $m$ can also be defined as a complex whose level- $n$ term is the free abelian group of the integral closed subschemes $Z \subset X \times \widetilde{B}_{n}$ which have the goodintersection property with all faces, and which satisfy the appropriate modulus condition on $X \times \widehat{B}_{n}$. The following lemma uses this particular definition of the additive cycle complex.

Lemma 5.5. Let $K$ be the function field of $\mathrm{SL}_{r+1, k}$, and $\phi: \square_{K}^{1} \rightarrow \mathrm{SL}_{r+1, K}$ be as in the previous lemma. Let $\mathrm{SL}_{r+1, K}$ act on $\mathbb{P}_{K}^{r}$ naturally. Consider the composition $H_{n}=p_{K / k} \circ \operatorname{pr}_{K}^{\prime} \circ \mu_{\phi}$ of morphisms

$$
\mathbb{P}^{r} \times \mathbb{A}^{1} \times \square_{K}^{n} \stackrel{\mu_{\phi}}{\longrightarrow} \mathbb{P}^{r} \times \mathbb{A}^{1} \times \square_{K}^{n} \stackrel{\mathrm{pr}_{K}^{\prime}}{\longrightarrow} \mathbb{P}^{r} \times \mathbb{A}^{1} \times \square_{K}^{n-1} \stackrel{p_{K / k}}{\longrightarrow} \mathbb{P}^{r} \times \mathbb{A}^{1} \times \square_{k}^{n-1},
$$

where

$$
\left\{\begin{array}{l}
\mu_{\phi}\left(x, t, y_{1}, \ldots, y_{n}\right):=\left(\phi\left(y_{1}\right) x, t, y_{1}, \ldots, y_{n}\right), \\
\operatorname{pr}_{K}^{\prime}\left(x, t, y_{1}, \ldots, y_{n-1}\right):=\left(x, t, y_{2}, \ldots, y_{n-1}\right), \\
p_{K / k}: \text { base change. }
\end{array}\right.
$$

Then for any $Z \in \mathrm{TZ}^{q}\left(\mathbb{P}_{k}^{r}, n ; m\right)$, the cycle $H_{n}^{*}(Z)=\mu_{\phi}^{*} \circ \operatorname{pr}_{K}^{\prime *}\left(Z_{K}\right)$ is admissible, hence it is in $\mathrm{TZ}^{q}\left(\mathbb{P}_{K}^{r}, n+1 ; m\right)$. Similarly, $H_{n}^{*}$ carries $\mathrm{TZ}_{\mathscr{W}}^{q}\left(\mathbb{P}_{k}^{r}, n ; m\right)$ to $\mathrm{TZ}_{W_{K}}^{q}\left(\mathbb{P}_{K}^{r}, n+1 ; m\right)$.

Proof. It is enough to prove the second assertion, that for any irreducible admissible $Z$ in $\mathrm{TZ}_{\mathscr{W}}^{q}\left(\mathbb{P}^{r}, n ; m\right)$, the variety $Z^{\prime}:=H_{n}^{*}(Z)$, that we informally call the "homotopy variety" of $Z$, satisfies the admissibility conditions of Definition 2.5.

Claim 1. The variety $Z^{\prime}$ intersects $W \times F_{K}$ properly for all $W \in W$ and for each face $F$ of $B_{n+1}$.

Proof. This follows from the arguments of [Bloch 1986, Lemma (2.2)] and [Levine 1998, Lemma 3.5.11] without any modification. We provide its proof for the sake of completeness. We may assume that $\mathcal{W}$ contains only one nonempty algebraic set $W$. There are two cases to consider.

Case 1. Suppose $F_{K}$ comes from $F=\mathbb{A}^{1} \times\{0\} \times F^{\prime}$ for some face $F^{\prime} \subset \square^{n-1}$. In this case, $Z^{\prime} \cap\left(W \times F_{K}\right)$ is nothing but $Z_{K} \cap\left(W \times \mathbb{A}^{1} \times F_{K}^{\prime}\right)$ because $\phi(0)=\operatorname{Id} \in$ $\mathrm{SL}_{r+1, K}$. So, proper intersection is obvious in this case. 
Case 2. Suppose $F_{K}$ does not come from faces of the form in Case 1. We apply Lemma 5.1 with $G=\mathrm{SL}_{r+1, k}, X=\mathbb{P}^{r} \times F, A=W \times F$, and $B=\operatorname{pr}_{k}^{\prime *}(Z) \cap\left(\mathbb{P}^{r} \times F\right)$, where $G$ acts on $X$ by acting trivially on $F$ and acting naturally on $\mathbb{P}^{r}$. By Lemma 5.1, there is a nonempty open subset $U \subset \mathrm{SL}_{r+1}$ such that for all $g \in U$, the intersection $g(A) \cap B$ is proper. By shrinking $U$ if necessary, we may assume that $U$ is invariant under taking the multiplicative inverses. Take $g=\eta^{-1} \in U$, the inverse of the generic point. Thus, after base extension to $K$, the intersection of $\eta^{-1}\left(W_{K} \times F_{K}\right)$ with $\operatorname{pr}_{K}^{\prime}{ }^{*}\left(Z_{K}\right) \cap\left(\mathbb{P}^{r} \times F_{K}\right)$ is proper, which means that $\eta\left(\operatorname{pr}_{K}^{\prime}{ }^{*}\left(Z_{K}\right) \cap\left(\mathbb{P}^{r} \times F_{K}\right)\right)$ intersects properly with $W_{K} \times F_{K}$. But the intersection $\mathrm{pr}_{K}^{\prime *}\left(Z_{K}\right) \cap\left(\mathbb{P}^{r} \times F_{K}\right)$ is proper, as $Z$ was admissible. Hence, $\eta\left(\mathrm{pr}_{K}^{\prime}{ }^{*}\left(Z_{K}\right)\right)$ intersects with $W_{K} \times F_{K}$ properly. Since $F$ is not of the form $\mathbb{A}^{1} \times\{0\} \times F^{\prime}, F_{K}$ intersects the first component $\square_{K}^{1}$ at $\{\infty\}$ nontrivially. In particular, $\eta\left(\operatorname{pr}_{K}^{\prime}{ }^{*}\left(Z_{K}\right)\right)$ is the same as $\mu_{\phi}^{*}\left(\operatorname{pr}_{K}^{\prime}{ }^{*}\left(Z_{K}\right)\right)=Z^{\prime}$ by Lemma 5.4. We conclude that $Z^{\prime}$ intersects with $W_{K} \times F_{K}$ properly. This proves the claim and hence $Z^{\prime}$ has the good-intersection property. Thus we only need to show the modulus condition for $Z^{\prime}$ to complete the proof of the lemma.

Claim 2. $Z^{\prime}$ satisfies the modulus condition on $\mathbb{P}^{r} \times \widetilde{B}_{n+1, K}$.

Proof. We prove this using our containment lemma. In the following, we casually drop the automorphism $\tau: \mathbb{P}^{r} \times \mathbb{A}^{1} \times \square^{n} \rightarrow \mathbb{P}^{r} \times \mathbb{A}^{1} \times \square^{n}$ that maps $\left(x, t, y_{1}, \ldots, y_{n}\right)$ to $\left(x, t, y_{2}, \ldots, y_{n}, y_{1}\right)$ from our notations for simplicity.

Take $V=p(Z)$, where $p: \mathbb{P}^{r} \times \widetilde{B}_{n} \rightarrow \widetilde{B}_{n}$ is the projection. Because $Z \subset$ $p^{-1}(p(Z))=\mathbb{P}^{r} \times V$, we have

$$
Z^{\prime}=\mu_{\phi}^{*}\left(Z \times \square_{K}^{1}\right) \subset \mu_{\phi}^{*}\left(\mathbb{P}^{r} \times V \times \square_{K}^{1}\right)=\mathbb{P}^{r} \times V \times \square_{K}^{1}=: Z_{1} \text {, say. }
$$

Now, Proposition 5.2 implies that $V$ is an irreducible admissible closed subvariety of $\widetilde{B}_{n}$. The flat pull-back property in turn implies that $p^{*}([V])=\mathbb{P}^{r} \times V$ is an irreducible admissible closed subvariety of $\mathbb{P}^{r} \times \widetilde{B}_{n}$. In particular, the modulus condition holds for $\mathbb{P}^{r} \times V$. If $\bar{V}$ is the closure of $V$ in $\widehat{B}_{n}$, then commutativity of the diagram

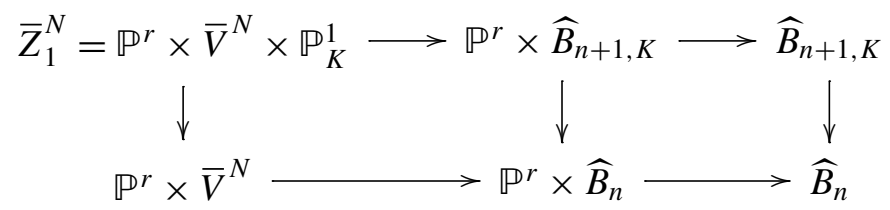

now implies that $Z_{1}$ satisfies the modulus condition on $\mathbb{P}^{r} \times \widetilde{B}_{n+1, K}$ even though it is a degenerate additive cycle. Furthermore, the admissibility of $Z$ and the fact that $\mu_{\phi}$ is an automorphism imply that $\bar{Z}^{\prime}$ intersects the Cartier divisors $F_{n+1}^{1}$ and $F_{n+1,0}$ properly. Thus we can use (5-1) and apply Proposition 2.4 to conclude that $Z^{\prime}$ satisfies the modulus condition. This completes the proof of the lemma. 
Lemma 5.6. The collection $H_{\bullet}^{*}: \mathrm{TZ}^{q}\left(\mathbb{P}_{k}^{r}, \cdot ; m\right) \rightarrow \mathrm{TZ}^{q}\left(\mathbb{P}_{K}^{r}, \cdot+1 ; m\right)$ is a chain homotopy satisfying $\partial H^{*}+H^{*} \partial=Z_{K}-\eta\left(Z_{K}\right)$. The same is true for $\mathrm{TZ}_{W}^{q}$.

Proof. It is enough to prove the second assertion. This is straightforward: let $Z \in \mathrm{TZ}_{\mathscr{W}}^{q}\left(\mathbb{P}_{k}^{r}, n ; m\right)$. Then

$$
\begin{aligned}
H^{*} \partial Z & =H^{*} \operatorname{sum}_{i=1}^{n-1}(-1)^{i}\left(\partial_{i}^{\infty}-\partial_{i}^{0}\right) Z=\operatorname{sum}_{i=1}^{n-1}(-1)^{i}\left(\mu_{\phi}^{*}\left(\operatorname{pr}_{K}^{\prime}\right)^{*} p_{K / k}^{*}\right)\left(\partial_{i}^{\infty}-\partial_{i}^{0}\right) Z \\
& =\operatorname{sum}_{i=1}^{n-1}(-1)^{i}\left(\partial_{i+1}^{\infty}-\partial_{i+1}^{0}\right) \mu_{\phi}^{*}\left(\operatorname{pr}_{K}^{\prime}\right)^{*} Z_{K}=-\operatorname{sum}_{i=2}^{n}(-1)^{i}\left(\partial_{i}^{\infty}-\partial_{i}^{0}\right) H^{*} Z, \\
\partial H^{*} Z & =\operatorname{sum}_{i=1}^{n}(-1)^{i}\left(\partial_{i}^{\infty}-\partial_{i}^{0}\right) H^{*} Z=\operatorname{sum}_{i=1}^{n}(-1)^{i}\left(\partial_{i}^{\infty}-\partial_{i}^{0}\right) H^{*} Z \\
& =(-1)\left(\partial_{1}^{\infty}-\partial_{1}^{0}\right) H^{*} Z+\operatorname{sum}_{i=2}^{n}(-1)^{i}\left(\partial_{i}^{\infty}-\partial_{i}^{0}\right) H^{*} Z .
\end{aligned}
$$

Hence, $\left(\partial H^{*}+H^{*} \partial\right) Z=\left(\partial_{1}^{0}-\partial_{1}^{\infty}\right) H^{*} Z=Z_{K}-\eta\left(Z_{K}\right)$.

5B. Proof of the moving lemma for projective spaces. We are now ready to finish the proof of Theorem 4.1 for $\mathbb{P}^{r}$.

By Lemma 5.6, the base extension

$$
p_{K / k}^{*}: \frac{\mathrm{TZ}^{q}\left(\mathbb{P}_{k}^{r}, \cdot ; m\right)}{\mathrm{TZ}_{W}^{q}\left(\mathbb{P}_{k}^{r}, \cdot ; m\right)} \rightarrow \frac{\mathrm{TZ}^{q}\left(\mathbb{P}_{K}^{r}, \cdot ; m\right)}{\mathrm{TZ}_{W_{K}}^{q}\left(\mathbb{P}_{K}^{r}, \cdot ; m\right)}
$$

is homotopic to the map $\eta p_{K / k}^{*}$. Note for each admissible cycle $Z \in \mathrm{TZ}^{q}\left(\mathbb{P}_{k}^{r}, n ; m\right)$, the cycle $\eta\left(Z_{K}\right)$ lies in $\mathrm{TZ}_{\mathscr{W}}^{q}\left(\mathbb{P}_{K}^{r}, n ; m\right)$. Part of the proof of Claim 1 of Lemma 5.5 is similar to the proof of this assertion:

We may assume that $\mathcal{W}$ has only one nonempty algebraic set, say $W$. Let $F$ be a face of $B_{n}$. In Lemma 5.1, take $G=\mathrm{SL}_{r+1}$ and $X=\mathbb{P}^{r} \times F$ where $G$ acts on $\mathbb{P}^{r}$ naturally and $B_{n}$ trivially. Let $A=W \times F$ and $B=Z \cap\left(\mathbb{P}^{r} \times F\right)$. Since $\mathrm{SL}_{r+1}$ acts transitively on $\mathbb{P}^{r}$, the map $G \times A \rightarrow X$ is surjective. Hence, by Lemma 5.1, there is a nonempty open subset $U \subset G$ such that for all $g \in U$, the intersection $g(A) \cap B$ is proper in $X$. By shrinking $U$ further, we may assume that $U$ is closed under taking multiplicative inverses. Taking $g=\eta^{-1}$, the inverse of the generic point, we see that after base extension to $K$, the intersection of $\eta^{-1}(W \times F)$ with $Z_{K} \cap\left(\mathbb{P}^{r} \times F_{K}\right)$ is proper, which means $\eta\left(Z_{K} \cap\left(\mathbb{P}^{r} \times F_{K}\right)\right)$ intersects $W_{K} \times F_{K}$ properly. Since $Z_{K}$ intersects with $\mathbb{P}^{r} \times F_{K}$ properly by the assumption, we conclude that $\eta\left(Z_{K}\right)$ intersects $W_{K} \times F_{K}$ properly. Thus, $\eta\left(Z_{K}\right) \in \mathrm{TZ}_{\mathscr{W}}^{q}\left(\mathbb{P}_{K}^{r}, n ; m\right)$. Hence, the induced map on the quotient

$$
\eta p_{K / k}^{*}: \frac{\mathrm{TZ}^{q}\left(\mathbb{P}_{k}^{r}, \cdot ; m\right)}{\mathrm{TZ}_{\mathscr{W}}^{q}\left(\mathbb{P}_{k}^{r}, \cdot ; m\right)} \rightarrow \frac{\mathrm{TZ}^{q}\left(\mathbb{P}_{K}^{r}, \cdot ; m\right)}{\mathrm{TZ}_{W_{K}}^{q}\left(\mathbb{P}_{K}^{r}, \cdot ; m\right)}
$$

is zero. Hence the base extension $p_{K / k}^{*}$ induces a zero map on homology since it is homotopic to the zero map.

On the other hand, by the spreading lemma, Proposition 4.7, the chain map $p_{K / k}^{*}$ is injective on homology, so the quotient complex $\mathrm{TZ}^{q}\left(\mathbb{P}_{k}^{r}, \cdot ; m\right) / \mathrm{TZ}_{W}^{q}\left(\mathbb{P}_{k}^{r}, \cdot ; m\right)$ must be acyclic. This proves Theorem 4.1 for the projective spaces. 


\section{Generic projections and moving lemma for projective varieties}

6A. Generic projections. This section begins with a review of some facts about linear projections. In combination with the moving lemma for $\mathbb{P}^{r}$, that we saw in the previous section, we prove the moving lemma for general smooth projective varieties.

Lemma 6.1. Consider two integers $N>r>0$. Then for each linear subvariety $L \subset \mathbb{P}^{N}$ of dimension $N-r-1$, there exists a linear projection morphism $\pi_{L}$ : $\mathbb{P}^{N} \backslash L \rightarrow \mathbb{P}^{r}$.

Proof. Fix the coordinates $x=\left(x_{0} ; \ldots ; x_{N}\right)$ of $\mathbb{P}^{N}$. A linear subvariety $L$ is given by $(r+1)$ homogeneous linear equations in $x$ whose corresponding $(N+1) \times(r+1)$ matrix $A$ has the full rank $r+1$. Take the reduced row echelon form of $A$ whose rows are the linear homogeneous functions $P_{0}(x), \ldots, P_{r}(x)$ in $x$.

For $x \in \mathbb{P}^{N} \backslash L$, define $\pi_{L}(x):=\left(P_{0}(x) ; \ldots ; P_{r}(x)\right)$. Since $x \notin L$, we have some $P_{i}(x) \neq 0$ so that the map $\pi_{L}$ is well-defined. By elementary facts about reduced row echelon forms and row equivalences, the subvariety $L$ uniquely decides this map $\pi_{L}$ in this process.

Let $X$ be a smooth projective $k$-variety. Let $r=\operatorname{dim} X$. Suppose that we have an embedding $X \hookrightarrow \mathbb{P}^{N}$ for some $N>r$. Consider $\pi_{L}: \mathbb{P}^{N} \backslash L \rightarrow \mathbb{P}^{r}$. Whenever $L \cap X=\varnothing$, we have a finite morphism $\pi_{L, X}:=\left.\pi_{L}\right|_{X}: X \rightarrow \mathbb{P}^{r}$. Such $L$ 's form a nonempty open subset $\operatorname{Gr}(N-r-1, N)_{X}$ of the Grassmannian $\operatorname{Gr}(N-r-1, N)$. Such a map $\pi_{L}$ is automatically flat since $X$ is smooth [Hartshorne 1977, Exercise III-10.9, p. 276]. In particular, the pull-back $\pi_{L, X}^{*}$ and push-forward $\pi_{L, X *}$ are defined by Theorem 3.1.

For any closed integral admissible cycle $Z$ on $X \times B_{n}$, define $\widetilde{L}(Z)$ to be

$$
\widetilde{L}(Z):=\pi_{L, X}^{*}\left(\pi_{L, X_{*}}([Z])\right)-[Z] .
$$

Extending this map linearly, this defines a morphism of complexes

$$
\widetilde{L}: \mathrm{TZ}^{q}(X, \cdot ; m) \rightarrow \mathrm{TZ}^{q}(X, \cdot ; m) .
$$

6B. Chow's moving lemma. Recall that for two locally closed subsets $A$ and $B$ of pure codimension $a$ and $b$, the excess of the intersection of $A$ and $B$ on $X$ is defined to be

$$
e(A, B):=\max \left\{a+b-\operatorname{codim}_{X}(A \cap B), 0\right\} .
$$

That the intersection $A \cap B$ is proper on $X$ means $e(A, B)=0$. If $A$ and $B$ are cycles, then we define $e(A, B):=e(\operatorname{Supp}(A), \operatorname{Supp}(B))$. The excess measures how far an intersection is from being proper.

Lemma 6.2 [Krishna and Levine 2008, Lemma 1.12]. Let $X \subset \mathbb{P}^{N}$ be a smooth closed projective $k$-subvariety of dimension $r$. Let $Z$ and $W$ be cycles on $X$. Then 
there is a nonempty open subscheme $U_{Z, W} \subset \operatorname{Gr}(N-r-1, N)_{X}$ such that for each field extension $K \supset k$ and each $K$-point $L$ of $U_{Z, W}$, we have

$$
e(\widetilde{L}(Z), W) \leq \max \{e(Z, W)-1,0\} .
$$

For its proof, see [Roberts 1972, Main Lemma, p. 93], or [Levine 1998, Lemma 3.5 .4 , p. 96] for a slightly different but equivalent version. The point of the projection business is the following lemma:

Lemma 6.3. Let $X$ be a smooth projective $k$-variety, and let $\mathcal{W}$ be a finite set of locally closed algebraic subsets of $X$. Let $m, N \geq 1$, and $q \geq 0$ be integers. Let $e: \mathcal{W} \rightarrow \mathbb{Z}_{\geq 0}$ be a set-theoretic function. Define $e-1: \mathcal{W} \rightarrow \mathbb{Z}_{\geq 0}$ by

$$
(e-1)(W):=\max \{e(W)-1,0\} .
$$

Let $K$ be the function field of $\operatorname{Gr}(N-r-1, N)$, and let $L_{\text {gen }} \in \operatorname{Gr}(N-r-1, N)_{X}(K)$ be the generic point. Then, the map

$$
\widetilde{L}_{\text {gen }}: \mathrm{TZ}^{q}(X, \cdot ; m) \rightarrow \mathrm{TZ}^{q}\left(X_{K}, \cdot ; m\right)
$$

maps $\mathrm{TZ}_{\mathscr{W}, e}^{q}(X, \cdot ; m)$ to $\mathrm{TZ}_{W_{K}, e-1}^{q}\left(X_{K}, \cdot ; m\right)$.

Proof. The arguments of [Krishna and Levine 2008, Lemma 1.13, p. 84] or [Levine 1998, \$3.5.6, p. 97] work in this additive context without change. The central idea is to use a variation of Chow's moving lemma as in Lemma 6.2.

\section{C. Proof of the moving lemma.}

Proof of Theorem 4.1. Let $L_{\mathrm{gen}}$ be the generic point of the Grassmannian $\operatorname{Gr}(N-$ $r-1, N)$ as in Lemma 6.3. Then, for each function $e: \mathcal{W} \rightarrow \mathbb{Z}_{\geq 0}$, the morphism

$$
\tilde{L}_{\text {gen }}=\pi_{L_{\text {gen }}}^{*} \circ \pi_{L_{\text {gen } *}}-p_{K / k}^{*}: \frac{\mathrm{TZ}_{W_{, e}}^{q}(X, \cdot ; m)}{\mathrm{TZ}_{\mathscr{W}, e-1}^{q}(X, \cdot ; m)} \rightarrow \frac{\mathrm{TZ}_{W_{K}, e}^{q}\left(X_{K}, \cdot ; m\right)}{\mathrm{TZ}_{W_{K}, e-1}^{q}\left(X_{K}, \cdot ; m\right)}
$$

is zero. Hence $\pi_{L_{\mathrm{gen}}}^{*} \circ \pi_{L_{\mathrm{gen}} *}$ is equal to the base extension morphism $p_{K / k}^{*}$ on the quotient complex.

On the other hand, $\pi_{L_{\text {gen }}}^{*} \circ \pi_{L_{\text {gen }} *}$ is written in detail as

$$
\frac{\mathrm{TZ}_{W_{, e}}^{q}(X, \cdot ; m)}{\mathrm{TZ}_{W_{, e-1}}^{q}(X, \cdot ; m)} \stackrel{\pi_{L \text { gen }} *}{\longrightarrow} \frac{\mathrm{TZ}_{W_{K}^{\prime}, e^{\prime}}^{q}\left(\mathbb{P}_{K}^{r}, \cdot ; m\right)}{\mathrm{TZ}_{W_{K}^{\prime}, e^{\prime}-1}^{q}\left(\mathbb{P}_{K}^{r}, \cdot ; m\right)} \stackrel{\pi_{L_{\text {gen }}^{*}}^{*}}{\longrightarrow} \frac{\mathrm{TZ}_{W_{K}, e}^{q}\left(X_{K}, \cdot ; m\right)}{\mathrm{TZ}_{W_{K}, e-1}^{q}\left(X_{K}, \cdot ; m\right)},
$$

where $\mathcal{W}^{\prime}$ and $e^{\prime}$ are defined as follows: for each $W \in \mathcal{W}$, the constructible subset $\pi_{L_{\text {gen }}}(W)$ can be written as

$$
\pi_{L_{\mathrm{gen}}}(W)=W_{1}^{\prime} \cup \cdots \cup W_{i_{W}}^{\prime}
$$

for some $i_{W} \in \mathbb{N}$ and locally closed irreducible sets $W_{j}^{\prime}$ in $\mathbb{P}_{K}^{r}$. Let $d_{j}=\operatorname{codim}_{\mathbb{P}_{K}^{n}}\left(W_{j}^{\prime}\right)$ $-\operatorname{codim}_{X}(W)$. Let $\mathcal{W}^{\prime}=\left\{W_{j}^{\prime} \mid W \in W\right\}$. Define $e^{\prime}: \mathscr{W}^{\prime} \rightarrow \mathbb{Z}_{\geq 0}$ by the rule 
$e^{\prime}\left(W_{j}^{\prime}\right):=e(W)+d_{j}$. We have already shown in Section 5B that the moving lemma is true for all projective spaces. In particular, for all functions $e^{\prime}:{ }^{\prime} W^{\prime} \rightarrow \mathbb{Z}_{\geq 0}$, the complex in the middle

$$
\frac{\mathrm{TZ}_{W_{K}^{\prime}, e^{\prime}}^{q}\left(\mathbb{P}_{K}^{r}, \cdot ; m\right)}{\mathrm{TZ}_{W_{K}^{\prime}, e^{\prime}-1}^{q}\left(\mathbb{P}_{K}^{r}, \cdot ; m\right)}
$$

is acyclic (see Remark 4.4). Hence, the base extension map

$$
p_{K / k}^{*}: \frac{\mathrm{TZ}_{\mathscr{W}, e}^{q}(X, \cdot ; m)}{\mathrm{TZ}_{\mathscr{W}, e-1}^{q}(X, \cdot ; m)} \rightarrow \frac{\mathrm{TZ}_{W_{K}, e}^{q}\left(X_{K}, \cdot ; m\right)}{\mathrm{TZ}_{W_{K}, e-1}^{q}\left(X_{K}, \cdot ; m\right)}
$$

is zero on homology. Consequently, by induction, the base extension map

$$
p_{K / k}^{*}: \frac{\mathrm{TZ}^{q}(X, \cdot ; m)}{\mathrm{TZ}_{\mathscr{W}}^{q}(X, \cdot ; m)} \rightarrow \frac{\mathrm{TZ}^{q}\left(X_{K}, \cdot ; m\right)}{\mathrm{TZ}_{W_{K}}^{q}\left(X_{K}, \cdot ; m\right)}
$$

is zero on homology. On the other hand, this map is also injective on homology by Proposition 4.7. This happens only when

$$
\frac{\mathrm{TZ}^{q}(X, \cdot ; m)}{\mathrm{TZ}_{W}^{q}(X, \cdot ; m)}
$$

is acyclic, i.e., the inclusion $\mathrm{TZ}_{\mathscr{W}}^{q}(X, \cdot ; m) \rightarrow \mathrm{TZ}^{q}(X, \cdot ; m)$ is a quasiisomorphism.

\section{Application to contravariant functoriality}

In this section, we prove the following general contravariance property of the additive higher Chow groups as an application of the moving lemma.

Theorem 7.1. Let $f: X \rightarrow Y$ be a morphism of quasiprojective varieties over $k$, where $Y$ is smooth and projective. Then there is a pull-back map

$$
f^{*}: \mathrm{TH}^{q}(Y, n ; m) \rightarrow \mathrm{TH}^{q}(X, n ; m)
$$

such that for a composition $X \stackrel{f}{\rightarrow} Y \stackrel{g}{\rightarrow} Z$ with $Y$ and $Z$ smooth and projective, we have

$$
(g \circ f)^{*}=f^{*} \circ g^{*}: \mathrm{TH}^{q}(Z, n ; m) \rightarrow \mathrm{TH}^{q}(X, n ; m) .
$$

Before proving this functoriality, we mention one more consequence of our containment lemma (Proposition 2.4).

Corollary 7.2. Let $X \stackrel{i}{\rightarrow} Y$ be a regular closed embedding of quasiprojective but not necessarily smooth varieties over $k$. Then there is a Gysin chain map of additive cycle complexes

$$
i^{*}: \mathrm{TZ}_{\{X\}}^{q}(Y, \cdot ; m) \rightarrow \mathrm{TZ}^{q}(X, \cdot ; m)
$$


Proof. Let $\iota: Z \subset Y \times B_{n}$ be a closed irreducible admissible subvariety in the group $\mathrm{TZ}_{\{X\}}^{q}(Y, n ; m)$. By assumption, $Z$ intersects all faces $X \times F$ properly. Hence the abstract intersection product of cycles $\left(X \times B_{n}\right) \cdot Z=\left[\iota^{*}\left(X \times B_{n}\right)\right] \in z^{q}\left(X \times B_{n}\right)$ is well-defined. Moreover, the intersection formula for the regular embedding implies that this intersection product commutes with the boundary maps [Fulton 1998, §2.3 and §6.3]. We want this cycle to be $i^{*}(Z)$. Thus we only need to show that each component of $Z \cap\left(X \times B_{n}\right)$ satisfies the modulus condition in order for $i^{*}$ to be a map of additive cycle complexes. Since $X \times \widehat{B}_{n}$ clearly intersects $F_{n}^{1}$ and $F_{n, 0}$ properly on $Y \times \widehat{B}_{n}$, this modulus condition follows directly from Proposition 2.4, for $Z$ has the modulus condition.

Proof of Theorem 7.1. We do this by imitating the proof of Theorem 4.1 in [Bloch 1986]. So, let $f: X \rightarrow Y$ be a map as in Theorem 7.1. Such a morphism can be factored as the composition

$$
X \stackrel{\mathrm{gr}_{f}}{\rightarrow} X \times Y \stackrel{\mathrm{pr}_{2}}{\rightarrow} Y
$$

where $\mathrm{gr}_{f}$ is the graph of $f$ and $\mathrm{pr}_{2}$ is the projection. Notice that $\mathrm{pr}_{2}$ is a flat map and moreover, the smoothness of $Y$ implies that $\mathrm{gr}_{f}$ is a regular closed embedding. Let $\Gamma_{f} \subset X \times Y$ denote the image of $\operatorname{gr}_{f}$ which is necessarily closed.

For $0 \leq i \leq \operatorname{dim} X$, let $Y_{i}$ be the Zariski closure of the collection of all points $y \in Y$ such that $\operatorname{dim} f^{-1}(y) \geq i$. We use the convention that $\operatorname{dim} \varnothing=-1$. Let $\mathscr{W}$ be the collection of the irreducible components of all $Y_{i}$. Then $\mathcal{W}$ is a finite collection.

Claim. Let $Z \in \mathrm{TZ}_{\mathscr{W}}^{q}(Y, n ; m)$ be an irreducible admissible closed subvariety of $Y \times B_{n}$. Then $\left(\mathrm{pr}_{2} \times \operatorname{Id}_{B_{n}}\right)^{-1}(Z)=X \times Z$ in $X \times Y \times B_{n}$ is an admissible closed subset that intersects $\Gamma_{f} \times F$ properly in $X \times Y \times B_{n}$ for all faces $F \subset B_{n}$. This gives a chain map

$$
\operatorname{pr}_{2}^{*}: \mathrm{TZ}_{\mathscr{W}}^{q}(Y, \cdot ; m) \rightarrow \mathrm{TZ}_{\left\{\Gamma_{f}\right\}}^{q}(X \times Y, \cdot, m) .
$$

That $\left(\mathrm{pr}_{2} \times \mathrm{Id}_{B_{n}}\right)^{-1}(Z)=X \times Z$ is admissible is obvious by [Krishna and Levine 2008, §3.4]. Since $Z$ intersects $W \times F$ properly for all $W \in \mathscr{W}$ and faces $F \subset B_{n}$, we have $\operatorname{dim} \widetilde{Z}_{i} \leq \operatorname{dim} Y_{i}+\operatorname{dim} F-q$, where $\widetilde{Z}_{i}:=Z \cap\left(Y_{i} \times F\right)$.

Now, $(X \times Z) \cap\left(\Gamma_{f} \times F\right)=\bigcup_{i} X \times \widetilde{Z}_{i}$, and for each $i$ we have $\operatorname{dim}\left(X \times \widetilde{Z}_{i}\right)=$ $\operatorname{dim} X+\operatorname{dim} \widetilde{Z}_{i} \leq \operatorname{dim} X+\operatorname{dim} F-q=\operatorname{dim}\left(\Gamma_{f} \times F\right)-q$. We conclude that $\operatorname{codim}_{\Gamma_{f} \times F}(X \times Z) \cap\left(\Gamma_{f} \times F\right) \geq q$, thus obtaining the desired map

$$
\operatorname{pr}_{2}^{*}: \mathrm{TZ}_{\mathscr{W}}^{q}(Y, n ; m) \rightarrow \mathrm{TZ}_{\left\{\Gamma_{f}\right\}}^{q}(X \times Y, n ; m)
$$

for each $n \geq 1$. That this gives a chain map is obvious since $f^{*}$ clearly commutes with the boundary maps. This proves the claim. 
The pull-back map $f^{*}$ is now given by composing pr $_{2}^{*}$ with the Gysin map gr* of Corollary 7.2 and then using the moving lemma, Theorem 4.1. The composition law can be checked directly from the construction of $f^{*}$. This completes the proof of Theorem 7.1.

\section{Remarks and computations}

8A. Moving modulus conditions. We saw that $M_{\text {sum }}$ and $M_{\text {ssup }}$ seem to have much better structural behavior than the modulus condition $M_{\text {sup }}$ of [Krishna and Levine 2008; Park 2009], and this makes the former better suited for being a motivic cohomology. On the other hand, in the main theorem of [Park 2009], the regulators on 1-cycles were defined with the modulus condition $M_{\text {sup. Although }}$ we have seen that this regulator map does exist and has good properties with the modulus condition $M_{\text {ssup }}$, its construction doesn't automatically generalize to the groups with $M_{\text {sum. }}$. So, one may ask the following.

Question 8.1. Given an $M_{\mathrm{sum}}$-admissible cycle $\xi$ with $\partial \xi=0$, can one find an

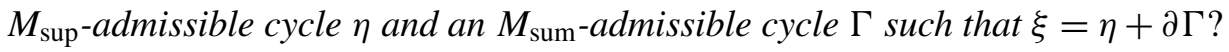

A positive answer to this question will immediately solve one part of Conjecture 2.8. This is a kind of deeper moving lemma than we have proved in this paper. This moving lemma allows one to move the modulus as well as the proper intersection property when we move a cycle. On the other hand, the moving lemma of this paper does not allow changing the modulus conditions. We expect the answer to the above question to be much harder.

\section{B. Examples.}

Example 8.2. We give a simple example where the homotopy used in [Bloch 1986; Levine 1998] doesn't preserve the modulus conditions for additive higher Chow groups of quasiprojective varieties.

Take $X=\mathbb{A}_{k}^{1}$ and $n=1$, so we are interested in admissible cycles in $X \times \widetilde{B}_{1}=$ $X \times \mathbb{A}_{k}^{1}$. Admissible closed subvarieties $Z \subset X \times \mathbb{A}_{k}^{1}$ are given by the condition $Z \cap(X \times\{0\})=\varnothing$. Let $\mathbb{G}_{a, k}=\mathbb{A}_{k}^{1}$ act on $X$ by translation, and take its function field $K=k(s), s$ transcendental over $k$. Take the line $\phi: \square_{K}^{1} \rightarrow \mathbb{G}_{a, K}$ defined by $y \mapsto s y /(y-1)$ that sends 0 to 0 and $\infty$ to the $k$-generic point $s$ of $\mathbb{G}_{a, k}$, which is $K$-rational in $\mathbb{G}_{a, K}$.

Take $Z$ given by the ideal $(x t+1) \subset k[x, t]$, which is in $\mathrm{TZ}^{1}\left(\mathbb{A}^{1}, 1 ; m\right)$. Then, $Z_{K}$ is given by $(x t+1) \subset K[x, t]$ and $\mathrm{pr}^{\prime *} Z_{K}$ is given by $(x t+1) \subset K[x, t, y /(y-1)]$. Pulling back through $\mu_{\phi}$, we get $(x+s y /(y-1)) t+1=0$. This is the equation for our homotopy variety $Z^{\prime}$. Rewriting it as $1-y=t((y-1) x+s y)$, we see that it doesn't satisfy any of the given modulus conditions $M_{\text {sum }}, M_{\text {sup }}, M_{\text {ssup }}$. For 
instance, for a given $m \geq 1$, we need $1-y$ to be divisible by at least $t^{1+m}$ where $m \geq 1$, which is obviously false in this case. Hence $Z^{\prime} \notin \mathrm{TZ}^{1}\left(\mathbb{A}_{K}^{1}, 2 ; m\right)$.

Example 8.3. Recall from Remark 5.3 that if $X$ is projective, then admissible cycles in $X \times \widetilde{B}_{1}=X \times \mathbb{A}^{1}$ have a very simple description: an admissible irreducible closed subvariety $Z$ should be of the form $Y \times\{*\} \subset X \times \mathbb{A}^{1}$ for some closed subvariety $Y \subset X$, and a closed point $\{*\} \neq\{0\}$ of $\mathbb{A}^{1}$. This variety obviously satisfies all of the modulus conditions.

Note that the admissible variety $Z$ in Example 8.2 is not of the form $Y \times\{*\}$ : this happens because $X=\mathbb{A}_{k}^{1}$ is not complete.

These two examples seem to suggest that one should possibly modify the definition of the additive higher Chow groups of a quasiprojective variety in such a way that it takes into account the behavior at infinity in any compactification of the underlying variety.

8C. A computation. We finish the paper with a calculation of some additive higher Chow groups, which the authors completed while working on this paper. The following extends [Bloch and Esnault 2003a, Theorem 6.4, p. 153] to affine spaces.

Theorem 8.4. Assume that $\frac{1}{6} \in k$. Let $M$ be a modulus condition $M_{\text {sum }}, M_{\text {sup }}$, or $M_{\text {ssup. }}$ Let $X=\mathbb{A}_{k}^{r}$, and let $m=1$. Then, the additive higher Chow groups of zero-dimensional cycles of $X$ are the absolute Kähler differentials of $k$ :

$$
\mathrm{TH}^{r+n}(X, n ; 1) \simeq \Omega_{k / \mathbb{Z}}^{n-1} .
$$

Remark 8.5. Note that, although it looks similar, this theorem does not imply that additive higher Chow groups have $\mathbb{A}^{1}$-homotopy invariance. For the structure morphism $\mathbb{A}_{k}^{r} \rightarrow \operatorname{Spec}(k)$, the pull-backs of 0-cycles on $\operatorname{Spec}(k) \times \widetilde{B}_{n}$ to $X \times \widetilde{B}_{n}$ are $r$-cycles, not 0 -cycles.

Proof. The proof is very similar to that of [Bloch and Esnault 2003a, Theorem 6.4, p. 153]. For a closed point $p \in X \times \widetilde{B}_{n}$ that does not intersect the faces and the divisor $\{t=0\}$, we define a homomorphism by setting

$$
\psi(p):=\operatorname{Tr}_{k(p) / k}\left(\frac{1}{t} \frac{d y_{1}}{y_{1}} \wedge \cdots \wedge \frac{d y_{n-1}}{y_{n-1}}\right)(p) \in \Omega_{k / \mathbb{Z}}^{n-1} .
$$

In other words, we ignore the coordinate of $X$. This defines a homomorphism $\psi: \operatorname{TZ}^{r+n}(X, n ; 1) \rightarrow \Omega_{k / \mathbb{Z}}^{n-1}$.

Claim 1. The composition

$$
\psi \circ \partial: \mathrm{TZ}^{r+n}(X, n+1 ; 1) \stackrel{\partial}{\rightarrow} \mathrm{TZ}^{r+n}(X, n ; 1) \stackrel{\psi}{\rightarrow} \Omega_{k / \mathbb{Z}}^{n-1}
$$

is zero.

Proof. This follows from [Bloch and Esnault 2003a, Proposition 6.2, p. 150]. 
Claim 2. Any two closed admissible points $p, p^{\prime} \in X \times \widetilde{B}_{n}$ for which only the coordinates of $X$ differ are equivalent as additive higher Chow cycles.

Proof. Note that the points $p, p^{\prime}$ are not assumed to be $k$-rational. Under the natural projections $\pi_{?}: X \times \widetilde{B}_{n} \rightarrow$ ?, where $?=X, \mathbb{A}^{1}$ and the $i$-th projection $\pi_{i}: X \times \widetilde{B}_{n} \rightarrow \square$, if one has $\pi_{X}(p)=a \in X, \pi_{\mathbb{A}^{1}}(p)=b \in \mathbb{A}^{1}$, and $\pi_{i}(p)=s_{i} \in \square$, for not necessarily $k$-rational closed points $a \in X, 0 \neq b \in \mathbb{A}^{1}, 0, \infty \neq s_{i} \in \square$, then one writes $p=\left(a, b, s_{1}, \ldots, s_{n-1}\right)$. Similarly, under the assumptions of Claim 2, one can write $p^{\prime}$ as $p^{\prime}=\left(a^{\prime}, b, s_{1}, \ldots, s_{n-1}\right)$, where $a^{\prime}$ is another closed point of $X$. Consider a parametrized line given in terms of the above notation,

$$
C=\left\{\left(a \frac{y}{y-1}+a^{\prime}\left(1-\frac{y}{y-1}\right), b, y, s_{1}, \ldots, s_{n-1}\right) \in X \times \widetilde{B}_{n+1} \mid y \in \square^{1}\right\},
$$

which is a closed 1-dimensional subvariety of $X \times \widetilde{B}_{n+1}$. This 1-cycle satisfies all the modulus conditions $M_{\text {sum }}, M_{\text {sup }}$, and $M_{\text {ssup }}$ having $b \neq 0$, and it intersects all faces properly having constant $y_{i}$-coordinate values $s_{i}$. Thus $C$ is admissible.

By direct calculations, $\partial_{1}^{0}(C)=p^{\prime}, \partial_{1}^{\infty}(C)=p$, and $\partial_{i}^{\epsilon}(C)=0$ for $i \geq 2$ and $\epsilon \in\{0, \infty\}$. Hence, $\partial(C)=p^{\prime}-p$ proving Claim 2 .

Given Claim 2, by [Bloch and Esnault 2003a, Proposition 6.3] and the rest of the arguments of [Bloch and Esnault 2003a, Theorem 6.4] for which $\frac{1}{6} \in k$ is used, the theorem follows.

We remark that the same arguments work for any variety $X$ as long as we can prove Claim 2. In particular, for any connected union of affine spaces, irreducible or not, we can conclude the same results.

\section{Acknowledgements}

The authors would like to thank Spencer Bloch, Hélène Esnault, and Marc Levine for their invaluable comments on the work. The authors feel very grateful to the anonymous referees who provided various suggestions that greatly improved many parts of this article.

Park would like to thank TIFR and KAIST for their hospitality and reduced teaching loads, and Juya for support throughout this work.

For this work, Park was partially supported by the Basic Science Research Program through the National Research Foundation of Korea (NRF) funded by the Ministry of Education, Science and Technology (2009-0063180).

\section{References}

[Bloch 1986] S. Bloch, "Algebraic cycles and higher K-theory", Adv. in Math. 61:3 (1986), 267304. MR 88f:18010 Zbl 0608.14004

[Bloch 1994] S. Bloch, "The moving lemma for higher Chow groups", J. Algebraic Geom. 3:3 (1994), 537-568. MR 96c:14007 Zbl 0830.14003 
[Bloch and Esnault 2003a] S. Bloch and H. Esnault, "The additive dilogarithm", pp. 131-155 in Documenta Mathematica, Kazuya Kato's Fiftieth Birthday, edited by S. e. a. Bloch, Documenta Mathematica, Bielefeld, Germany, 2003. Extra Vol. MR 2005e:19006 Zbl 1052.11048

[Bloch and Esnault 2003b] S. Bloch and H. Esnault, "An additive version of higher Chow groups", Ann. Sci. École Norm. Sup. (4) 36:3 (2003), 463-477. MR 2004c:14035 Zbl 1100.14014

[Chow 1956] W.-L. Chow, "On equivalence classes of cycles in an algebraic variety", Ann. of Math. 64 (1956), 450-479. MR 18,509a Zbl 0073.37304

[Fulton 1998] W. Fulton, Intersection theory, 2nd ed., Ergebnisse der Mathematik und ihrer Grenzgebiete 2, Springer, Berlin, 1998. MR 99d:14003 Zbl 0885.14002

[Guillén and Navarro Aznar 2002] F. Guillén and V. Navarro Aznar, "Un critère d'extension des foncteurs définis sur les schémas lisses”, Publ. Math. Inst. Hautes Études Sci. 95 (2002), 1-91.

[Hanamura 2004] M. Hanamura, "Mixed motives and algebraic cycles, II", Invent. Math. 158:1 (2004), 105-179. MR 2005g:14021 Zbl 1068.14022

[Hartshorne 1977] R. Hartshorne, Algebraic geometry, Graduate Texts in Mathematics 52, Springer, New York, 1977. MR 57 \#3116 Zbl 0367.14001

[Krishna and Levine 2008] A. Krishna and M. Levine, "Additive higher Chow groups of schemes", J. Reine Angew. Math. 619 (2008), 75-140. MR 2009d:14005 Zbl 1158.14009

[Krishna and Park 2011] A. Krishna and J. Park, "DGA-structure on additive higher Chow groups", preprint, 2011, available at http://mathsci.kaist.ac.kr/ jinhyun/papers/CDGA.pdf.

[Krishna and Park 2012] A. Krishna and J. Park, "Mixed motives over $k[t] /\left(t^{m+1}\right)$ ", J. Inst. Math. Jussieu 11:3 (2012), 611-657.

[Levine 1998] M. Levine, Mixed motives, Mathematical Surveys and Monographs 57, American Mathematical Society, Providence, RI, 1998. MR 99i:14025 Zbl 0902.14003

[Loday 1998] J.-L. Loday, Cyclic homology, 2nd ed., Grundlehren der Math. Wissenschaften 301, Springer, Berlin, 1998. MR 98h:16014 Zbl 0885.18007

[Park 2007] J. Park, "Algebraic cycles and additive dilogarithm", Int. Math. Res. Not. 2007:18 (2007). MR 2008i:14015

[Park 2009] J. Park, "Regulators on additive higher Chow groups", Amer. J. Math. 131:1 (2009), 257-276. MR 2009k:14012 Zbl 1176.14001

[Roberts 1972] J. Roberts, "Chow's moving lemma”, Appendix 2 to "Motives”, by S. L. Kleiman, pp. 53-82 in Algebraic geometry (Oslo, 1970), edited by F. Oort, Wolters-Noordhoff, Groningen, 1972. MR 52 \#3152 Zbl 0285.14005

[Rülling 2007] K. Rülling, "The generalized de Rham-Witt complex over a field is a complex of zero-cycles”, J. Algebraic Geom. 16:1 (2007), 109-169. MR 2007j:14006 Zbl 1122.14006

Communicated by Hélène Esnault

Received 2010-05-30 Revised 2011-01-09 Accepted 2011-02-06

amal@math.tifr.res.in School of Mathematics,

Tata Institute of Fundamental Research,

Homi Bhabha Road, Colaba, Mumbai-400005, India

jinhyun@mathsci.kaist.ac.kr Department of Mathematical Sciences,

Korea Advanced Institute of Science and Technology,

291 Daehak-ro, Yuseong-gu, Daejeon 305-701, South Korea 


\section{Algebra \& Number Theory}

msp.berkeley.edu/ant

\section{EDITORS}

MANAGING EDITOR

Bjorn Poonen

Massachusetts Institute of Technology

Cambridge, USA

\author{
EDITORIAL BOARD CHAIR \\ David Eisenbud \\ University of California \\ Berkeley, USA
}

\section{BOARD OF EDITORS}

Georgia Benkart

Dave Benson

Richard E. Borcherds

John H. Coates

J-L. Colliot-Thélène

Brian D. Conrad

Hélène Esnault

Hubert Flenner

Edward Frenkel

Andrew Granville

Joseph Gubeladze

Ehud Hrushovski

Craig Huneke

Mikhail Kapranov

Yujiro Kawamata

János Kollár

Yuri Manin

Barry Mazur

Philippe Michel

Susan Montgomery
University of Wisconsin, Madison, USA

University of Aberdeen, Scotland

University of California, Berkeley, USA

University of Cambridge, UK

CNRS, Université Paris-Sud, France

University of Michigan, USA

Universität Duisburg-Essen, Germany

Ruhr-Universität, Germany

University of California, Berkeley, USA

Université de Montréal, Canada

San Francisco State University, USA

Hebrew University, Israel

University of Kansas, USA

Yale University, USA

University of Tokyo, Japan

Princeton University, USA

Northwestern University, USA

Harvard University, USA

École Polytechnique Fédérale de Lausanne

University of Southern California, USA
Shigefumi Mori

Raman Parimala

Jonathan Pila

Victor Reiner

Karl Rubin

Peter Sarnak

Joseph H. Silverman

Michael Singer

Ronald Solomon

Vasudevan Srinivas

J. Toby Stafford

Bernd Sturmfels

Richard Taylor

Ravi Vakil

Michel van den Bergh

Marie-France Vignéras

Kei-Ichi Watanabe

Andrei Zelevinsky

Efim Zelmanov
RIMS, Kyoto University, Japan

Emory University, USA

University of Oxford, UK

University of Minnesota, USA

University of California, Irvine, USA

Princeton University, USA

Brown University, USA

North Carolina State University, USA

Ohio State University, USA

Tata Inst. of Fund. Research, India

University of Michigan, USA

University of California, Berkeley, USA

Harvard University, USA

Stanford University, USA

Hasselt University, Belgium

Université Paris VII, France

Nihon University, Japan

Northeastern University, USA

University of California, San Diego, USA

\section{PRODUCTION}

contact@msp.org

Silvio Levy, Scientific Editor

See inside back cover or www.jant.org for submission instructions.

The subscription price for 2012 is US \$175/year for the electronic version, and \$275/year (+\$40 shipping outside the US) for print and electronic. Subscriptions, requests for back issues from the last three years and changes of subscribers address should be sent to Mathematical Sciences Publishers, Department of Mathematics, University of California, Berkeley, CA 94720-3840, USA.

Algebra \& Number Theory (ISSN 1937-0652) at Mathematical Sciences Publishers, Department of Mathematics, University of California, Berkeley, CA 94720-3840 is published continuously online. Periodical rate postage paid at Berkeley, CA 94704, and additional mailing offices.

ANT peer review and production are managed by EditFLOW ${ }^{\circledR}$ from Mathematical Sciences Publishers.

PUBLISHED BY

mathematical sciences publishers

http://msp.org/

A NON-PROFIT CORPORATION

Typeset in IAT $_{\mathrm{E}} \mathrm{X}$

Copyright (C2012 by Mathematical Sciences Publishers 


\section{Algebra \& Number Theory}

Volume $6 \quad$ No. $2 \quad 2012$

Arithmetic of singular Enriques surfaces

Klaus HuleK and MatThias SchÜtT

An upper bound on the Abbes-Saito filtration for finite flat group schemes and applications

YICHAO TIAN

On the smallest number of generators and the probability of generating an algebra

Rostyslav V. Kravchenko, Marcin Mazur and Bogdan V. Petrenko

Moving lemma for additive higher Chow groups

AMALENDU KRISHNA and JINHYUN PARK

CHRISTOPHER GOFF

Uniformly rigid spaces

CHRISTIAN KAPPEN

On a conjecture of Kontsevich and Soibelman

LÊ QUY THUONG 